\title{
Investigations on Performance Metrics of FINFET based 8- Bit Low Power Adder Architectures Implemented using Various Logic Styles
}

\author{
M. Aalelai Vendhan* and S. Deepa \\ Department of Electronics and Communication Engineering, Panimalar Engineering College, Chennai - 600123, \\ Tamil Nadu, India; aalelai07@gmail.com, dineshdeepas1977@gmail.com
}

\begin{abstract}
Objectives: To reduce the leakage power dissipation and minimize the propagation delay, a Fin FET based 8-bit adder architecture is constructed. The performance metrics of these structures are calculated over a range of temperatures and are compared with the MOSFET based 8-bit adder architecture. Various logic styles are utilized for constructing the adder. Method/Analysis: A Ripple carry adder structure is employed. The existing adder architecture is constructed using 90nm MOSFET technology. The proposed adder architecture is constructed using $32 \mathrm{~nm}$ FINFET technology. The various logic styles employed are Complementary Metal-Oxide Semiconductor logic (CMOS), Complementary Pass-Transistor Logic (CPL), Transmission Gate logic (TG) and Gate Diffusion Input logic (GDI). Cadence Virtuoso is used for designing purpose and simulation is performed using Spectre. Findings: Key performance metrics like static power, dynamic power, leakage power, delay and power delay product are calculated. The dynamic power of MOSFET architecture ranges from $7.42 \mu \mathrm{W}$ to $882.6 \mu \mathrm{W}$. The dynamic power of FINFET architecture ranges from $0.407 \mathrm{nW}$ to $156.2 \mathrm{nW}$. The static power (inputs at high logic level) of MOSFET architecture ranges from $0.001 \mu \mathrm{W}$ to $945.76 \mu \mathrm{W}$. The static power (inputs at high logic level) of Fin FET architecture ranges from $0.725 \mathrm{pW}$ to $170.4 \mathrm{nW}$. The static power (inputs at low logic level) of MOSFET architecture ranges from $0.94 \mathrm{nW}$ to $1.68 \mathrm{~mW}$. The static power (inputs at low logic level) of Fin FET architecture ranges from $0.127 \mathrm{pW}$ to $305.3 \mathrm{nW}$. The leakage power (inputs at high logic level) of MOSFET architecture ranges from $1.27 \mathrm{nW}$ to $134.7 \mu \mathrm{W}$. The leakage power (inputs at high logic level) of Fin FET architecture ranges from $0.36 \mathrm{pW}$ to $24.77 \mathrm{nW}$. The leakage power (inputs at low logic level) of MOSFET architecture ranges from $0.54 \mathrm{nW}$ to $139.9 \mu \mathrm{W}$. The leakage power (inputs at low logic level) of Fin FET architecture ranges from $0.15 \mathrm{nW}$ to $227.6 \mathrm{nW}$. The delay of MOSFET architecture ranges from $0.344 \mu$ s to $0.46 \mu$ s. The delay of Fin FET architecture ranges from $0.19 \mu$ s to $0.28 \mu$ s. The power delay product of MOSFET architecture ranges from 2.66 to 405.99 . The power delay product of Fin FET architecture ranges from 0.83 to 29.67. Novelty/Improvements: The FINFET adder architecture proved to be effective in reducing the propagation delay and leakage power dissipation. This may find usage in high performance devices like microchips and supercomputers.
\end{abstract}

Keywords: Complementary Pass-transistor Logic, Gate Diffusion Input, Low Power Adder, Transmission Gate, CMOS, FINFET

\section{Introduction}

In recent years, the leakage power has become predominant power dissipation due to the scaling of the feature size to sub nanometer level. As the device feature size is scaled down, the threshold voltages are also scaled down to a minimum level, which exponentially increases the

${ }^{*}$ Author for correspondence 
leakage during the standby mode especially when all NMOS devices are in cut-off state. Hence, it is mandatory to minimize the leakage power during the cut-off state of the transistors. Fin FET transistors are used to minimize the leakage power since the multi-gate structure of the Fin FET minimizes the leakage power during cut-off state.

Many techniques have been employed to overcome power dissipation issues. A novel technique of constructing such an adder is described in $\stackrel{1}{1}$, where different logic styles like CMOS, CPL, TG are employed for designing a 1-bit FINFET added. Comparatively, the proposed TG-based full adder was the most successful design. The TG-based adder had the least power consumption. A similar technique was used in ${ }^{2}$ where a 1-bit adder was designed using a Double Gate (DG) Fin FET at $45 \mathrm{~nm}$ technology. It was observed that the adder designed using DG Fin FET has shown leakage power reduction from $1.594 \mathrm{pW}$ to $1.09 \mathrm{pW}$ and leakage current reduction from $652.4 \mathrm{n}$ A to $1.71 \mathrm{n} \mathrm{A}$ as compared with the CMOS 1-bit full adder cell. An ultra-low power 32-bit adder was designed in ${ }^{3}$ which made use of a $45 \mathrm{~nm}$ Fin FET. In this study, the dynamic energy and static energy show an inverse behavior with the increase in supply voltage. This adder consumed only $10 \mathrm{fj}$ for a single operation. The supply voltage provided was less than $100 \mathrm{mV}$. A 1-bit full adder technique using Fin FET technology is presented in $^{4}$ where various performance metrics like dissipation power, Power Delay Product (PDP) and Energy Delay Product (EDP) were calculated. The proposed Fin FET architecture provided $97 \%$ decrease in power dissipation and $99 \%$ reduction in PDP and EDP.

Another pioneering adder technique is employed in $^{\underline{5}}$ where a $32 \mathrm{~nm}$ Fin FET structure is used and the circuit is designed using Cell Design Methodology (CDM). CDM technique employs less number of transistors in the critical path. These adders have a delay reduction of $54 \%$ to $95 \%$, power delay product reduction of $33 \%$ to $76 \%$, energy efficiency is improved from $78 \%$ to $84 \%$. A 1-bit full adder cell is investigated in ${ }^{-6}$ where a GDI cell and DG structures are used. DG structures help in overcoming the effects of short channel effects. Independent Gate Control method is used in the circuit. GDI technique helps in the minimizing the number of transistors deployed. The proposed adder dissipated a power of $0.529 \mu \mathrm{W}$ and it had a delay of $32.82 \mathrm{ps} . \mathrm{In}^{\mathrm{Z}}$ various cell design like mirror adder, $14 \mathrm{~T}$ adder, TG based adder and adder using pass transistor were simulated. The number of transistors in mirror adder is reduced from 14 to 10 and the proposed structure also has $25 \%$ reduced leakage power. The leakage current was significantly reduced using $14 \mathrm{~T}$ added. The leakage current was reduced by a factor of 6 by using the TG model of adder. In pass transistor adder, the leakage current was reduced by a factor of 17 . Compared to all the models, $14 \mathrm{~T}$ adder was highly recommended.

Taking into consideration of the above, this paper contributes to the following:

- Realize 8-bit Ripple Carry Adder in 90nm MOSFET and 32nm Fin FET technology.

- Various logic styles like CPL, CMOS, TG, and GDI are employed.

- Performance metrics like Dynamic power, Static power, Leakage power, and Delay and Power delay product are measured.

\section{Simulation Results and Discussion}

The performance metrics are measured for every $25^{\circ} \mathrm{C}$ increase in temperature $\left(-25^{\circ} \mathrm{Cto} 125^{\circ} \mathrm{C}\right)$. The width of a PMOS transistor in MOSFET adder is $480 \mathrm{~nm}$. The width of a NMOS transistor in MOSFET adder is $120 \mathrm{~nm}$. The length of both PMOS and NMOS transistor in MOSFET structure is $100 \mathrm{~nm}$. The supply voltage is provided with $1 \mathrm{~V}$. The width of a PMOS transistor in Fin FET adder is $100 \mathrm{~nm}$. The width of a NMOS transistor in Fin FET adder is $50 \mathrm{~nm}$. The length of both PMOS and NMOS transistor in MOSFET structure is $32 \mathrm{~nm}$. The supply voltage is provided with $500 \mathrm{mV}$.

The Figure 1 depicts the schematic of 8-bit MOSFET adder implemented in CMOS logic. The Figure 2 repre- 


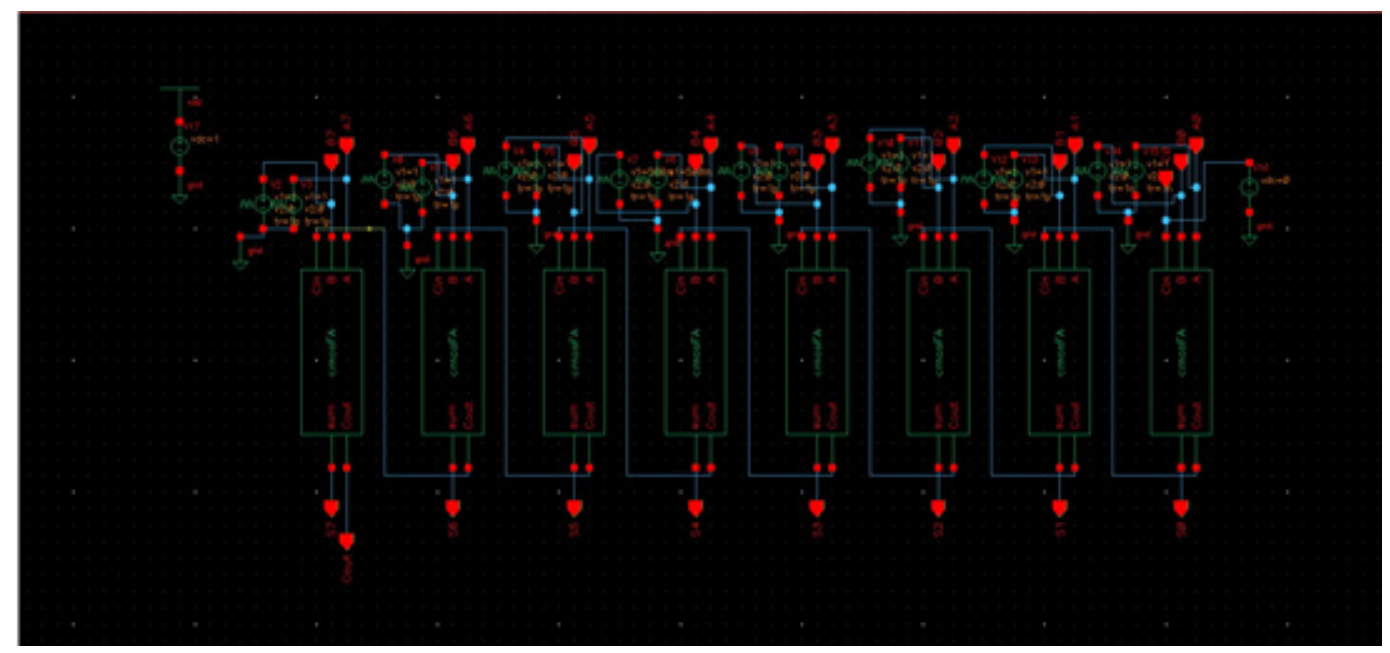

Figure 1. Schematic of 8-bit MOSFET adder in CMOS logic.

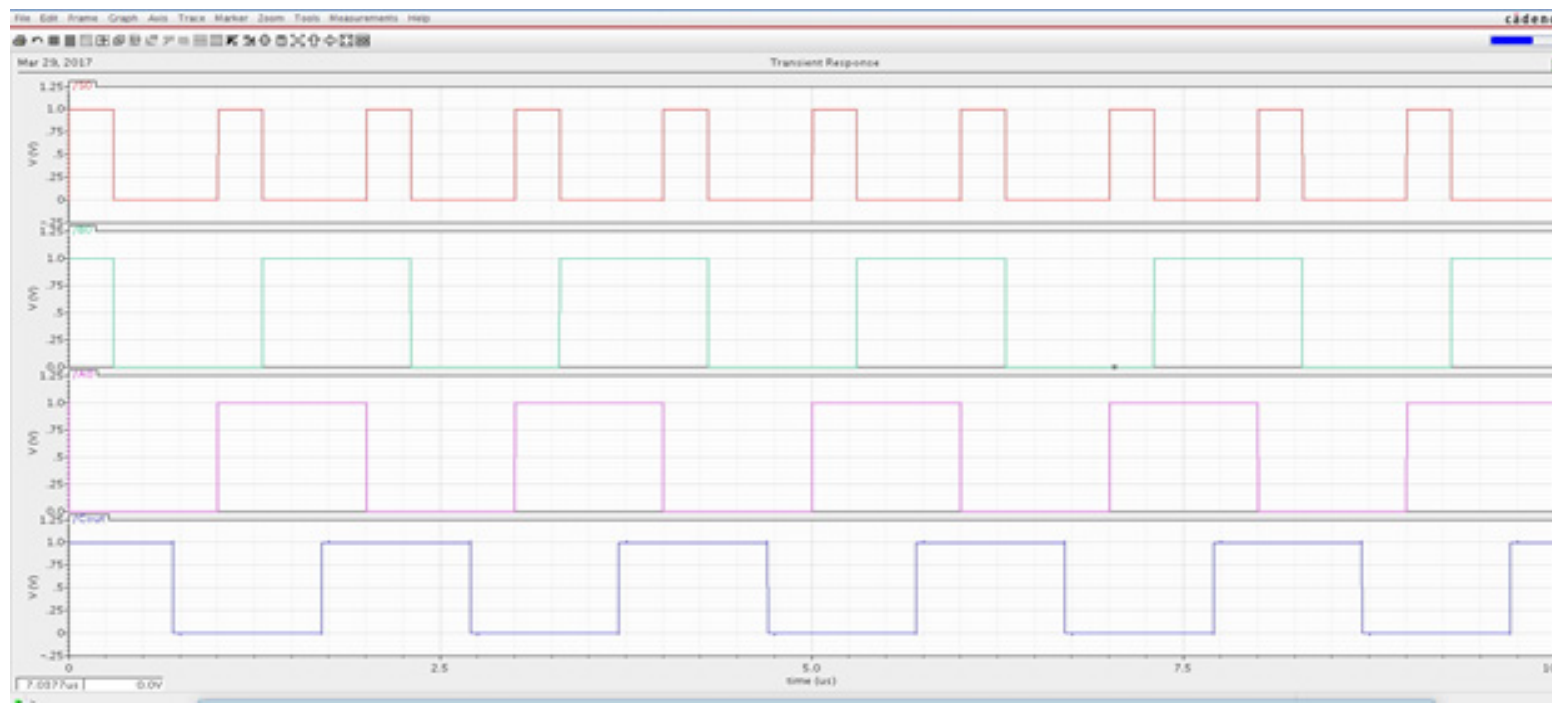

Figure 2. I/O waveform of 8-bit MOSFET adder in CMOS logic.

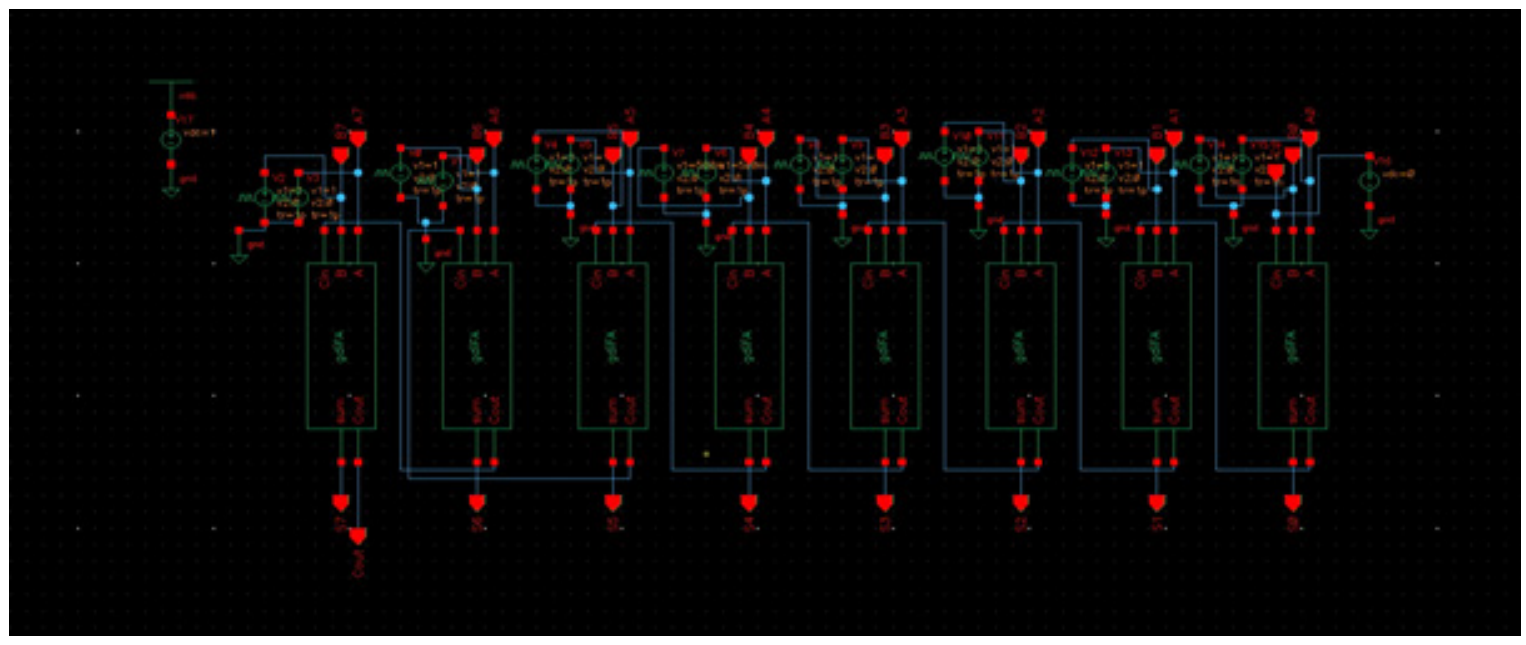

Figure 3. Schematic of 8-bit MOSFET adder in GDI logic. 


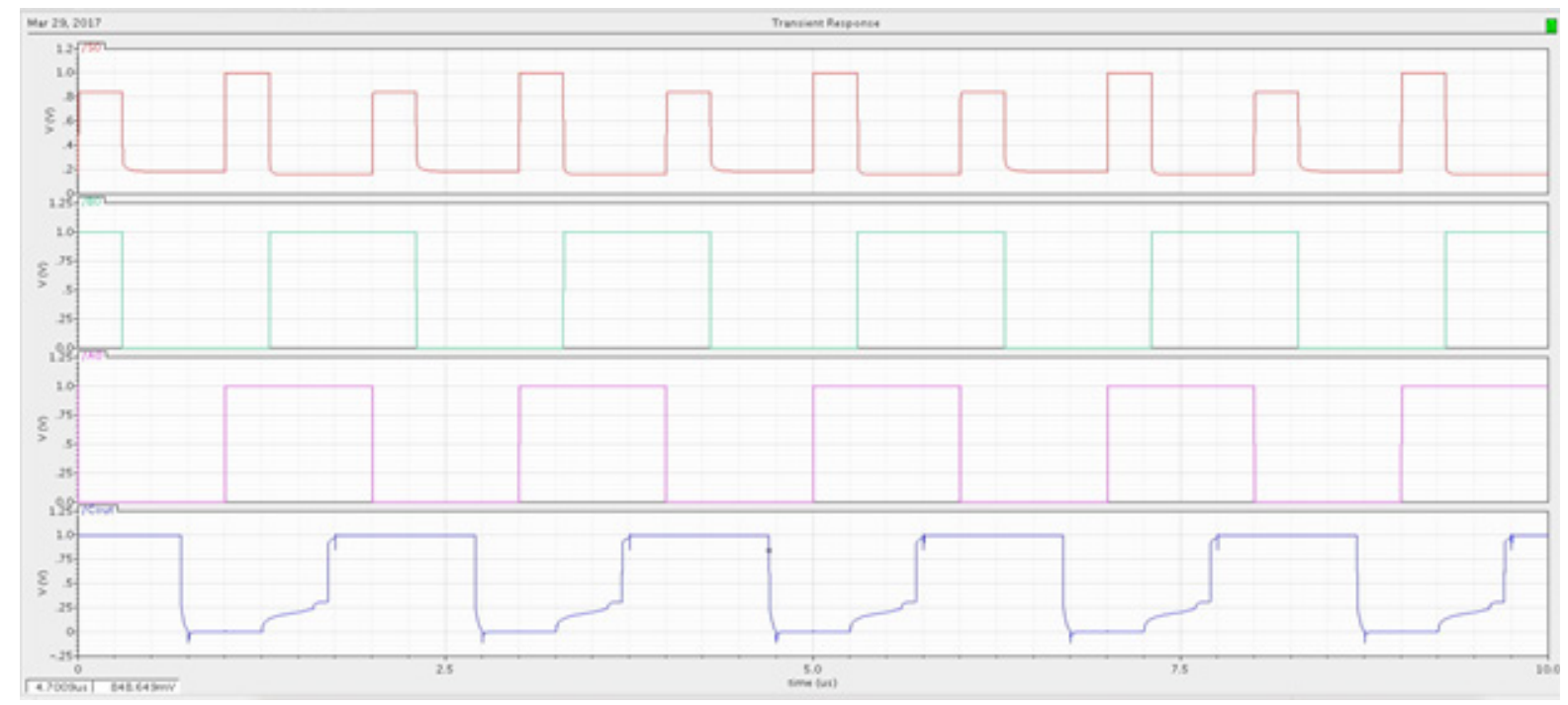

Figure 4. I/O waveform of 8-bit MOSFET adder in GDI logic.

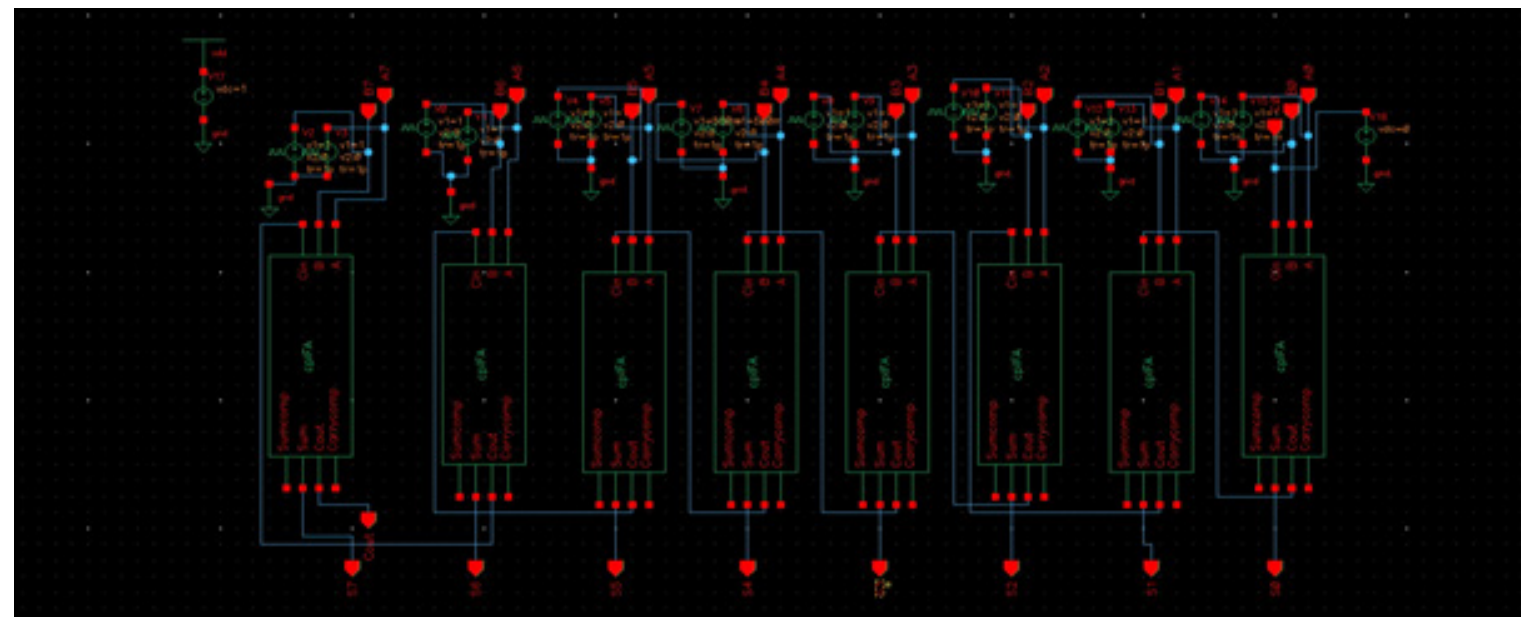

Figure 5. Schematic of 8-bit MOSFET adder in CPL logic.

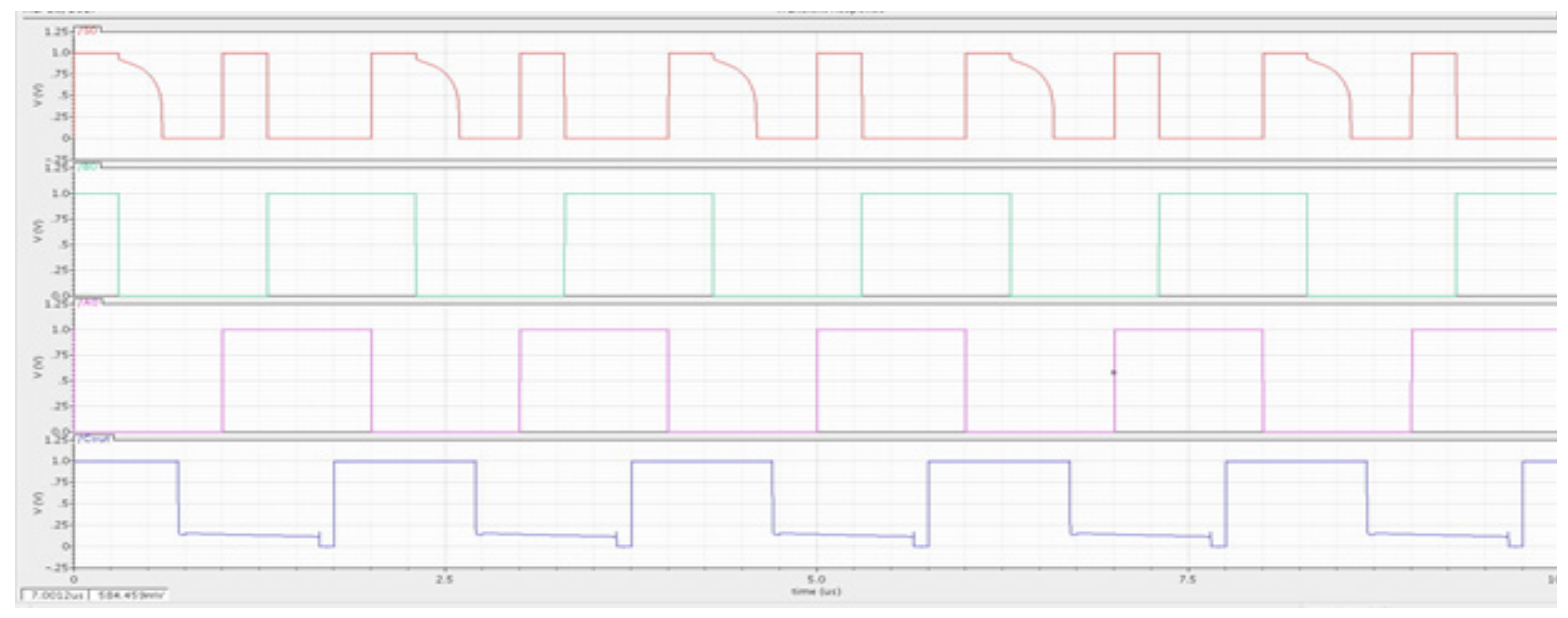

Figure 6. I/O waveform of 8-bit MOSFET adder in CPL logic. 


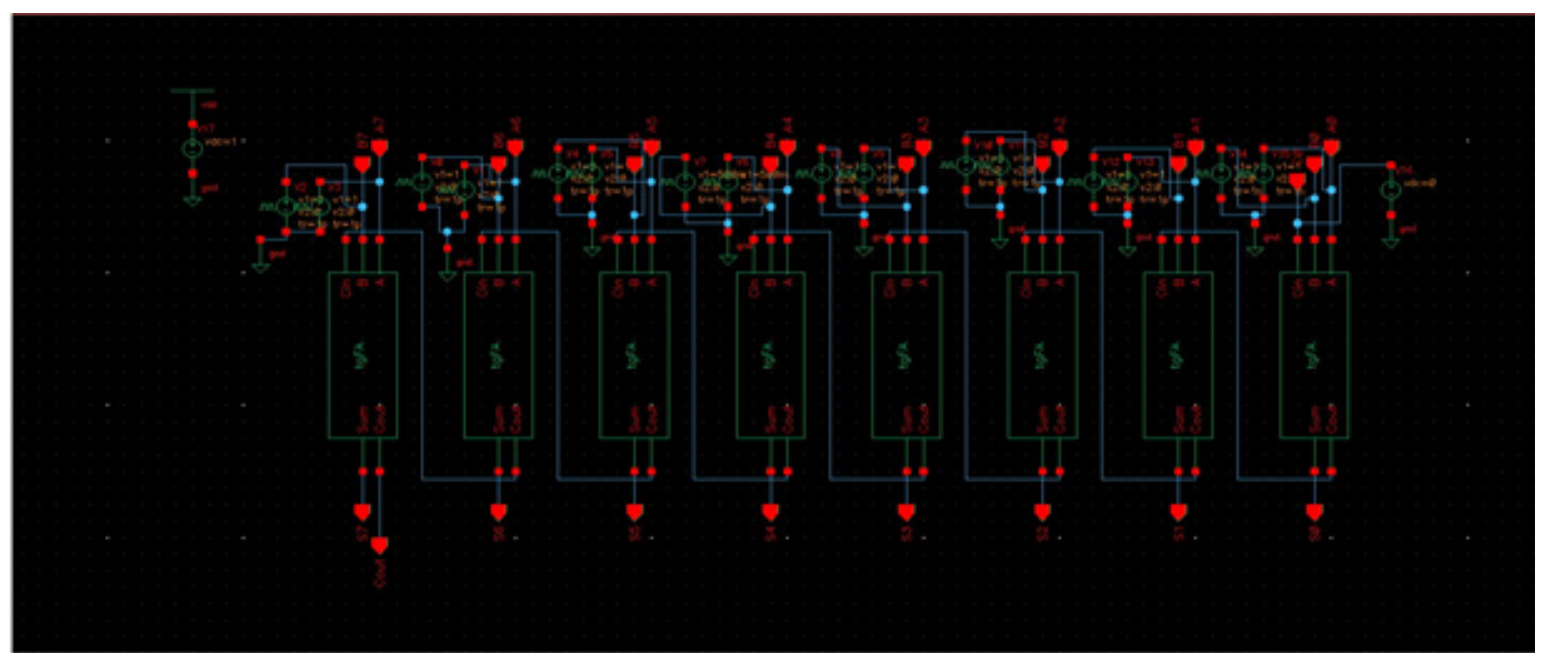

Figure 7. Schematic of 8-bit MOSFET adder in TG logic.

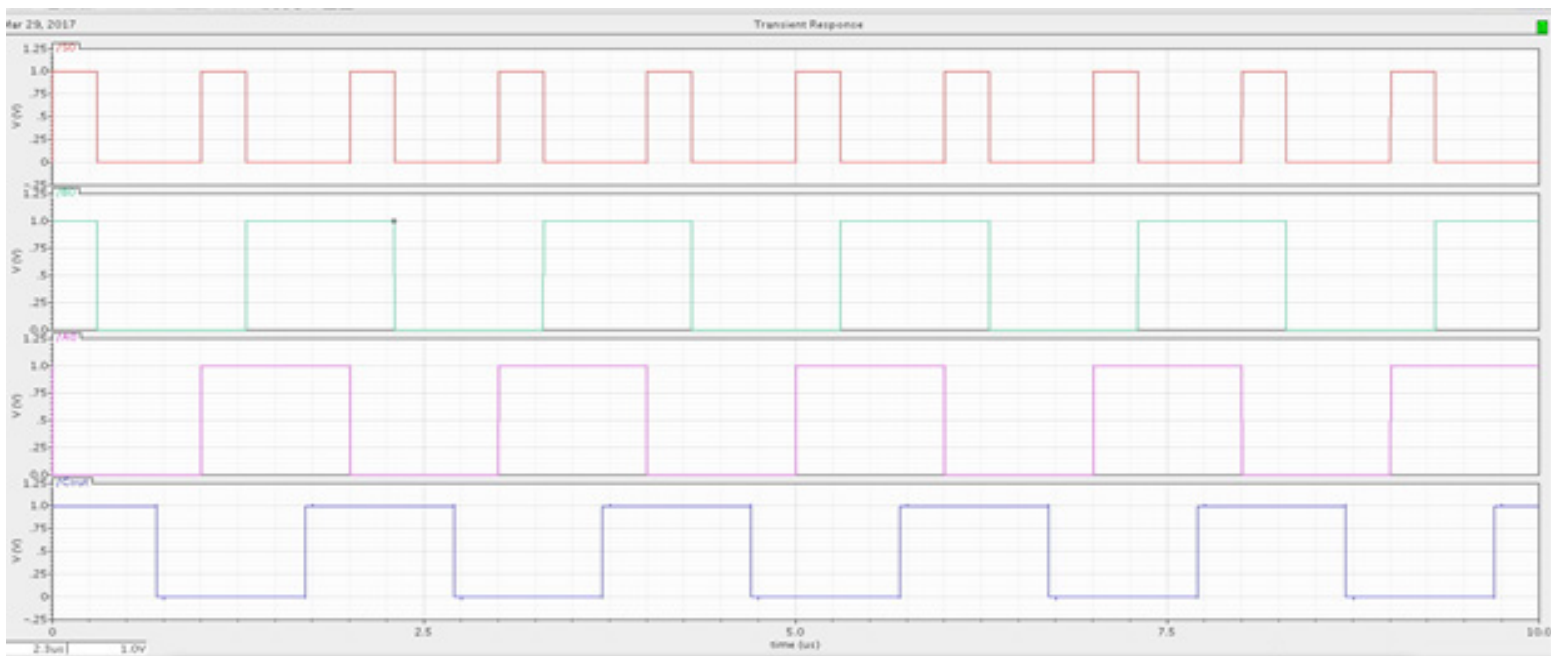

Figure 8. I/O waveform of 8-bit MOSFET adder in TG logic.

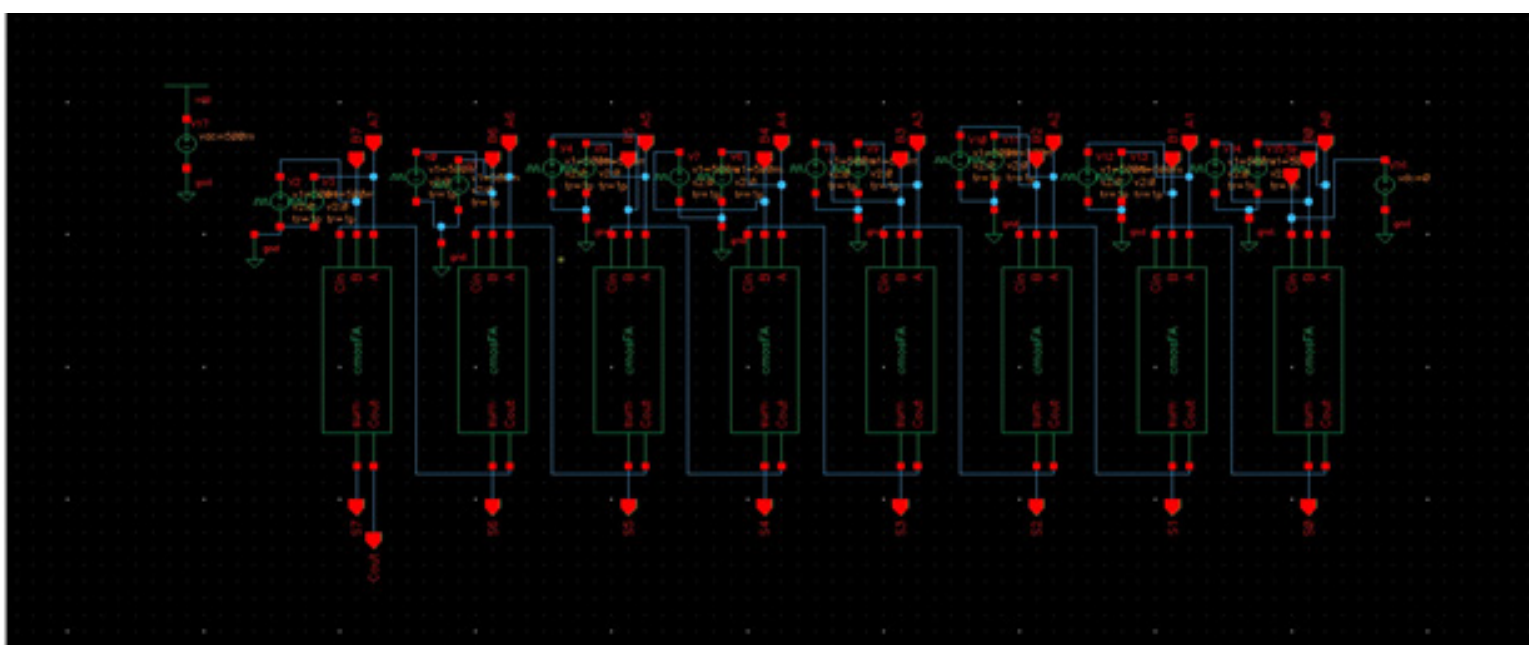

Figure 9. Schematic of 8-bit FinFET adder in CMOS logic. 


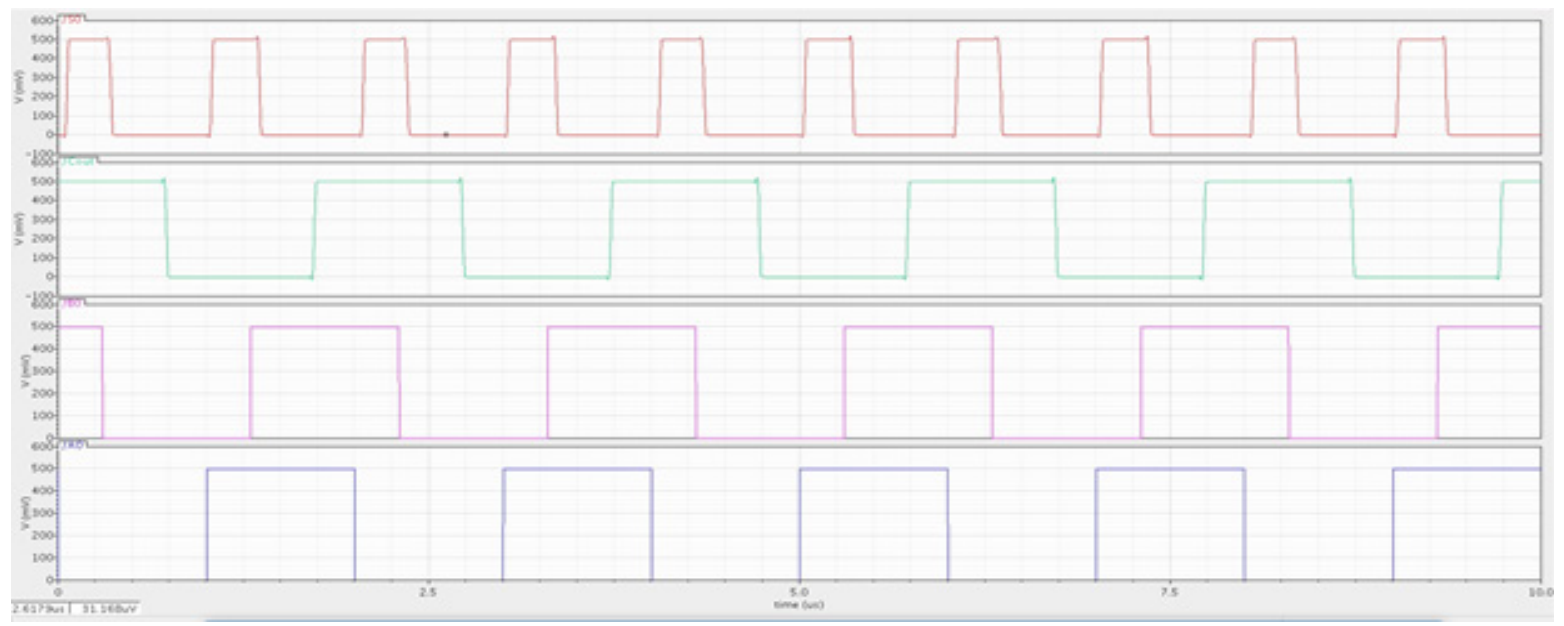

Figure 10. I/O waveform of 8-bit FinFET adder in CMOS logic.

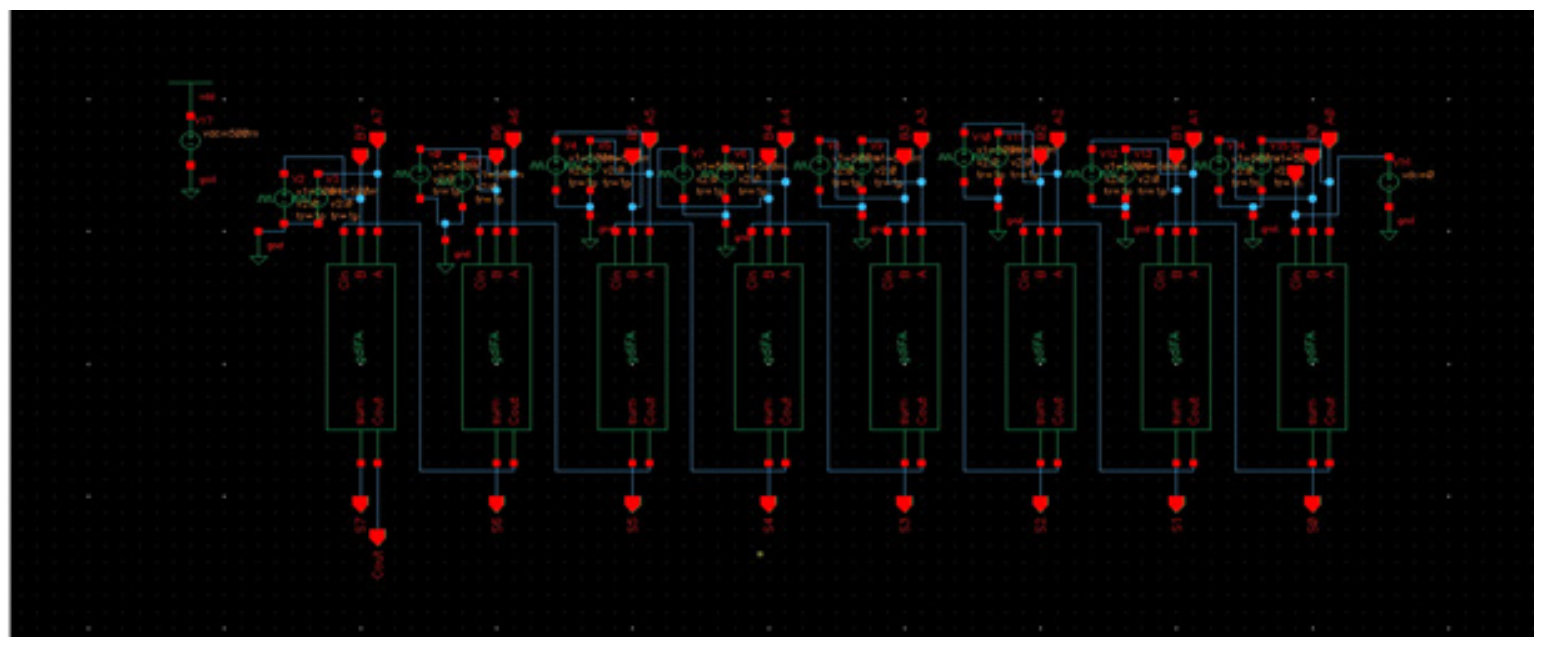

Figure 11. Schematic of 8-bit FinFET adder in GDI logic.

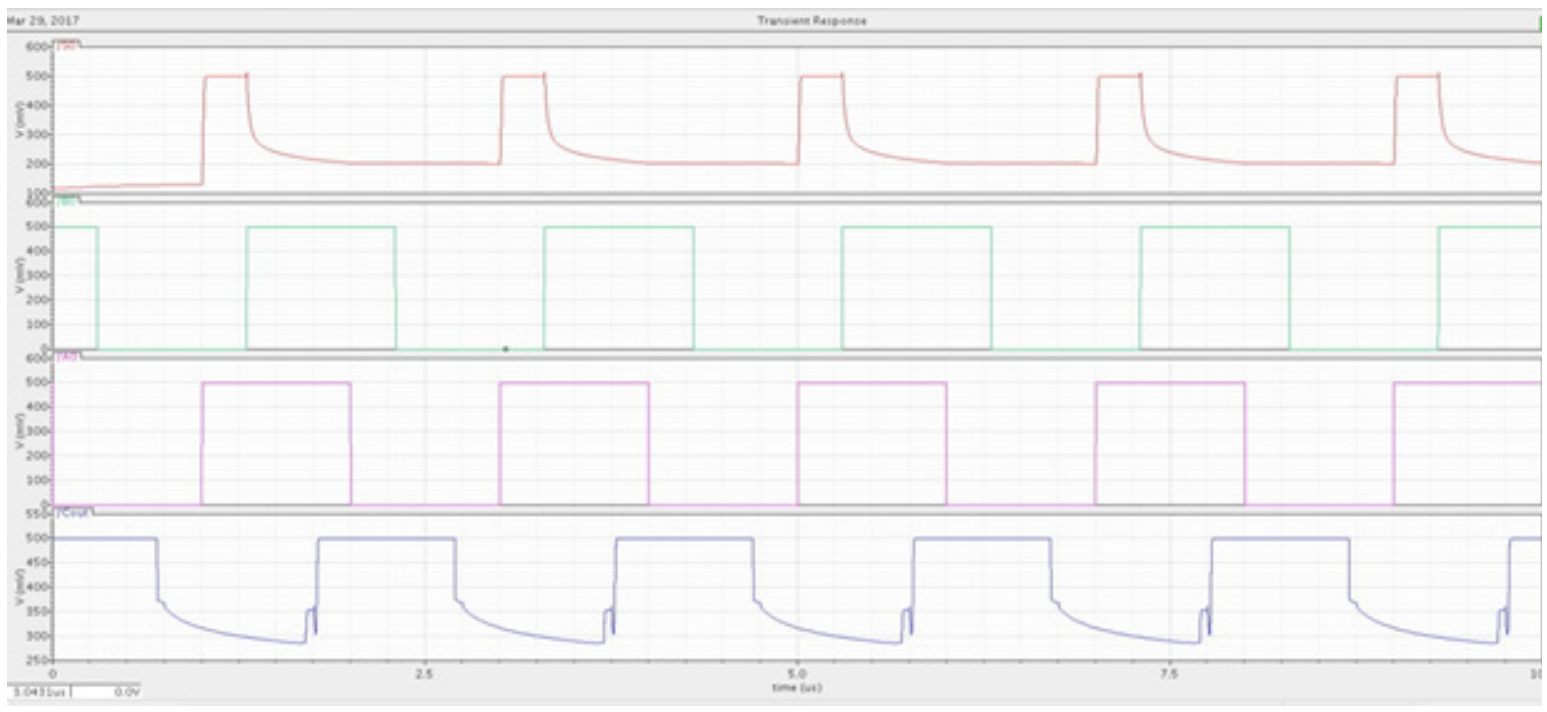

Figure 12. I/O waveform of 8-bit FinFET adder in GDI logic. 


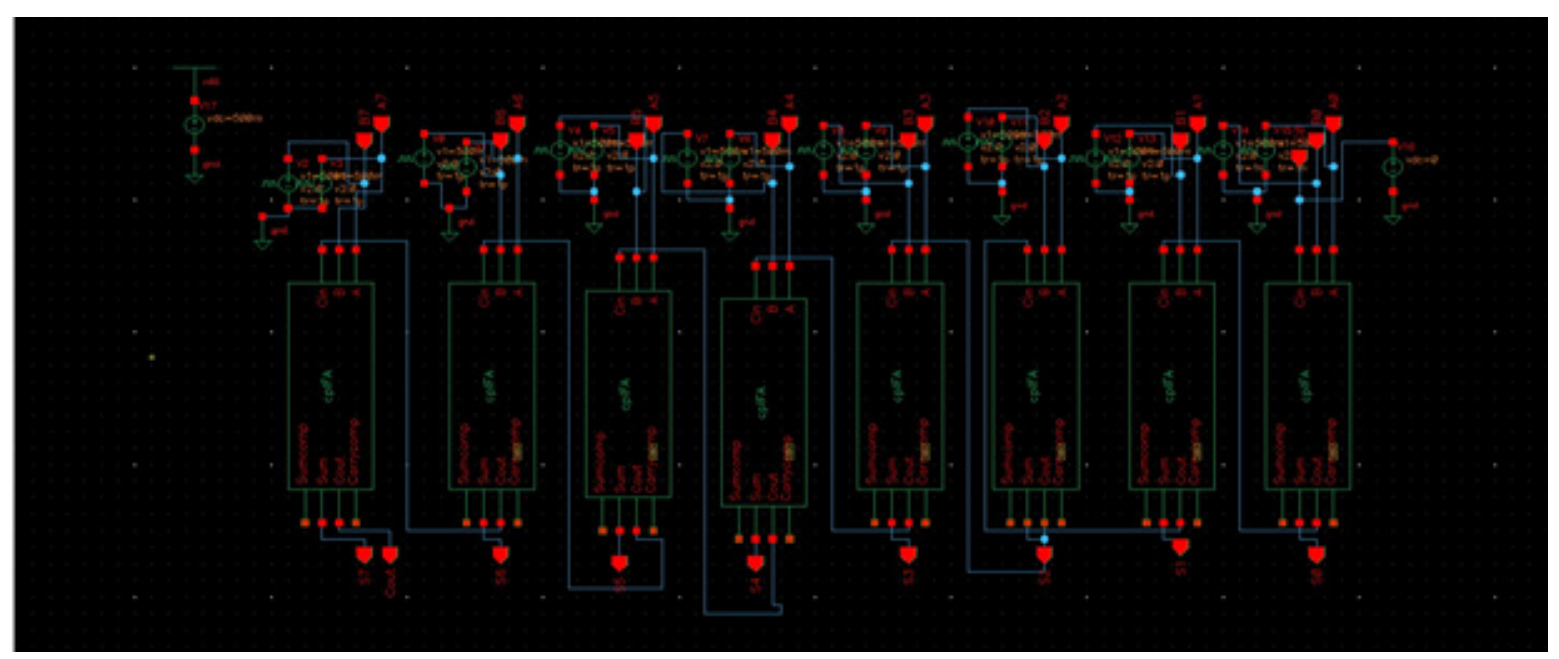

Figure 13. Schematic of 8-bit FinFET adder in CPL logic.

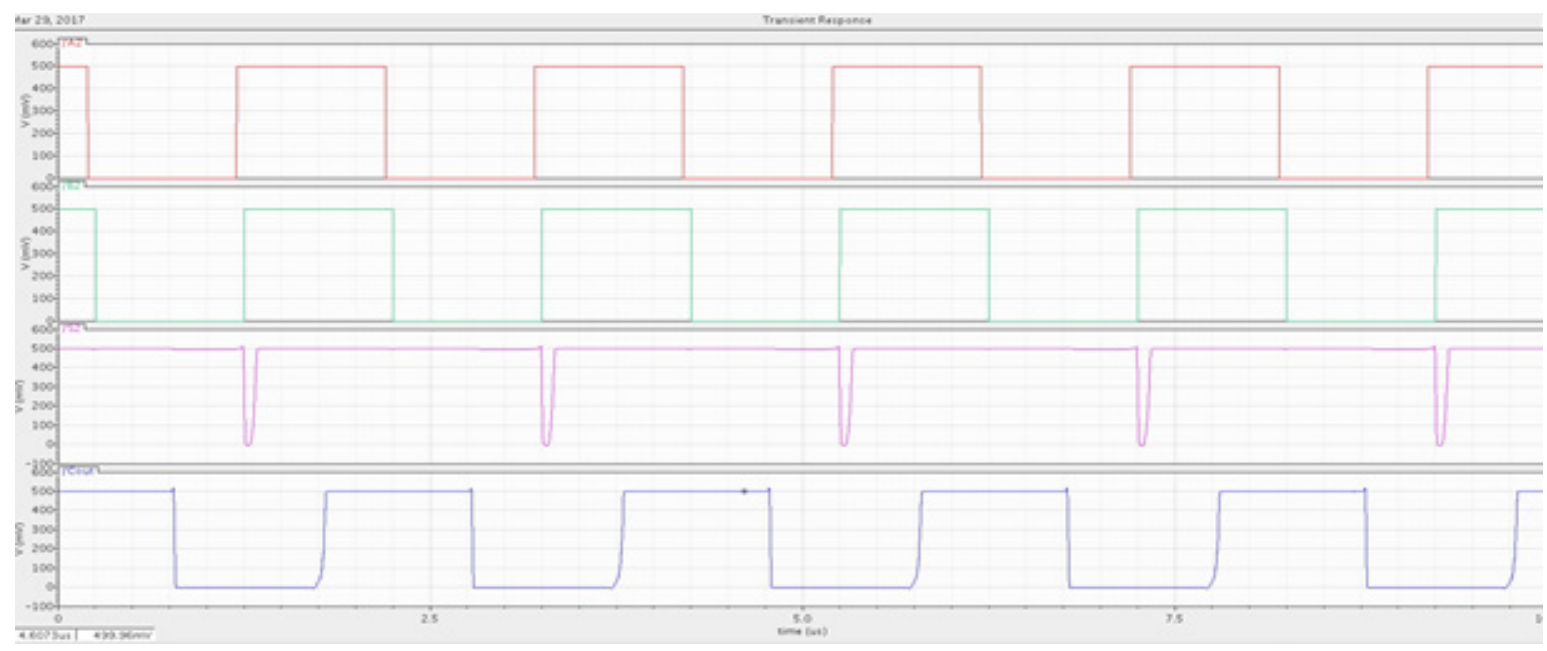

Figure 14. I/O waveform of 8-bit FinFET adder in CPL logic.

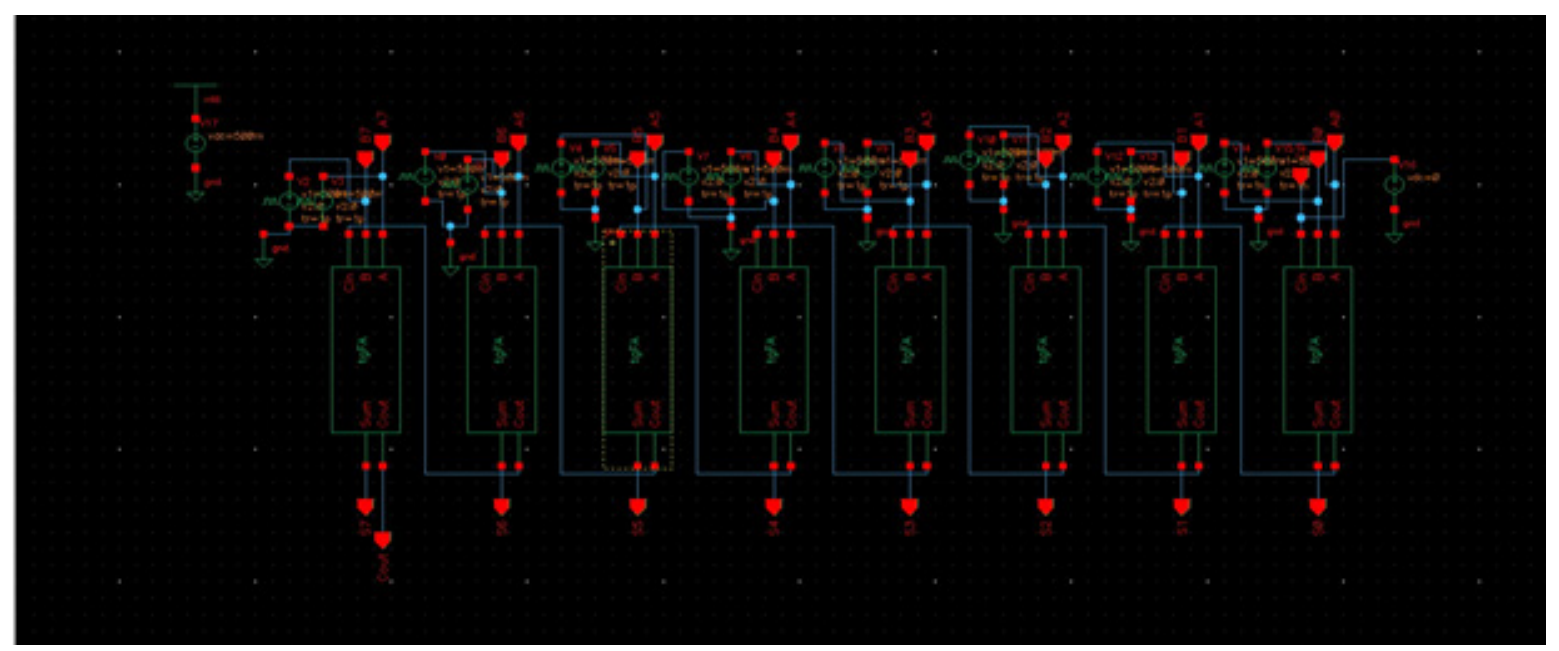

Figure 15. Schematic of 8-bit FinFET adder in TG logic. 


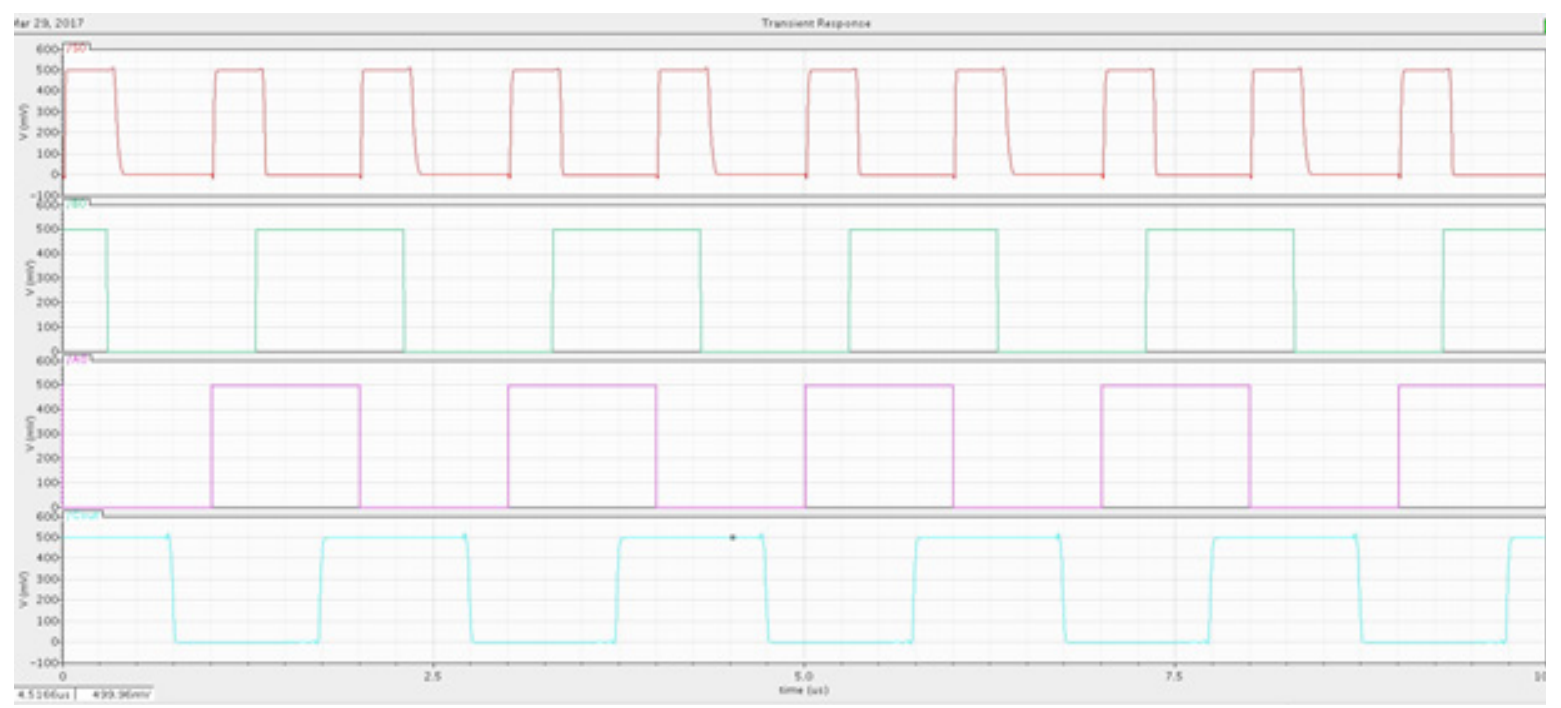

Figure 16. I/O waveform of 8-bit FinFET adder in TG logic.

Table 1. Performance metrics of 8-bit MOSFET adder in CMOS logic

\begin{tabular}{|c|c|c|c|c|c|c|c|}
\hline Temperature & All 0's & All 1's & & \\
\hline & $\begin{array}{c}\text { Dynamic } \\
\text { Power }\end{array}$ & $\begin{array}{c}\text { Static } \\
\text { Power }\end{array}$ & $\begin{array}{c}\text { Leakage } \\
\text { Power }\end{array}$ & $\begin{array}{c}\text { Static } \\
\text { Power }\end{array}$ & $\begin{array}{c}\text { Leakage } \\
\text { Power }\end{array}$ & Delay & $\begin{array}{c}\text { Power Delay } \\
\text { Product }\end{array}$ \\
\hline$($ Celsius $)$ & $(\mu \mathrm{W})$ & $(\mu \mathrm{W})$ & $(\mu \mathrm{W})$ & $(\mu \mathrm{W})$ & $(\mu \mathrm{W})$ & $(\mu \mathrm{s})$ & \\
\hline-25 & 6.41 & 0.469 & 2.42 & 0.001 & 3.601 & 0.41 & 2.62 \\
\hline 0 & 8.76 & 0.613 & 4.071 & 0.0125 & 5.481 & 0.404 & 3.54 \\
\hline 25 & 11.86 & 1.14 & 5.79 & 0.081 & 7.67 & 0.4 & 4.63 \\
\hline 50 & 15.03 & 1.56 & 8.143 & 0.187 & 10.17 & 0.399 & 5.99 \\
\hline 100 & 23.33 & 2.88 & 14.25 & 0.313 & 16.47 & 0.389 & 9.07 \\
\hline
\end{tabular}


sents the Input / Output (I/O) waveform of this adder. Table 1 contains the information about variation of the performance metrics of this adder in accordance to the range of temperatures. Figure 3 depicts the schematic of 8-bit MOSFET adder implemented in GDI logic. The Figure 4 represents the Input / Output (I/O) waveform of this adder. Table 2 contains the information about variation of the performance metrics of this adder in accordance to the range of temperatures. Figure 5 depicts the schematic of 8-bit MOSFET adder implemented in CPL logic. The Figure 6 represents the Input / Output (I/O) waveform of this adder. Table 3 contains the information about variation of the performance metrics of this adder in accordance to the range of temperatures. Figure 7 depicts the schematic of 8-bit MOSFET adder imple- mented in TG logic. The Figure 8 represents the Input / Output (I/O) waveform of this adder. Table 4 contains the information about variation of the performance metrics of this adder in accordance to the range of temperatures. Figure 9 depicts the schematic of 8-bit Fin FET adder implemented in CMOS logic. The Figure 10 represents the Input / Output (I/O) waveform of this adder. Table 5 contains the information about variation of the performance metrics of this adder in accordance to the range of temperatures. Figure 11 depicts the schematic of 8-bit Fin FET adder implemented in GDI logic. The Figure 12 represents the Input / Output (I/O) waveform of this adder. Table 6 contains the information about variation of the performance metrics of this adder in accordance to the range of temperatures. Figure 13 depicts the schematic of

Table 2. Performance metrics of 8-bit MOSFET adder in GDI logic

\begin{tabular}{|c|c|c|c|c|c|c|c|}
\hline Temperature & All 0's & & All 1's & & \\
\hline & $\begin{array}{c}\text { Dynamic } \\
\text { Power }\end{array}$ & $\begin{array}{c}\text { Static } \\
\text { Power }\end{array}$ & $\begin{array}{c}\text { Leakage } \\
\text { Power }\end{array}$ & $\begin{array}{c}\text { Static } \\
\text { Power }\end{array}$ & $\begin{array}{c}\text { Leakage } \\
\text { Power }\end{array}$ & Delay & $\begin{array}{c}\text { Power Delay } \\
\text { Product }\end{array}$ \\
\hline$($ Celsius $)$ & $(\mu \mathbf{W})$ & $(\mathbf{n W})$ & $(\mu \mathbf{W})$ & $(\mu \mathbf{W})$ & $(\mathbf{n W})$ & $(\boldsymbol{\mu s})$ & \\
\hline-25 & 13.68 & 0.94 & 0.54 & 0.024 & 1.27 & 0.344 & 4.71 \\
\hline 0 & 10.36 & 0.23 & 0.82 & 0.032 & 4.01 & 0.346 & 3.58 \\
\hline 25 & 8.59 & 1.17 & 1.15 & 0.049 & 10.83 & 0.35 & 3.01 \\
\hline 50 & 7.72 & 6.83 & 1.53 & 0.086 & 24.97 & 0.351 & 2.702 \\
\hline 125 & 7.42 & 25.68 & 3.01 & 0.447 & 157.8 & 0.359 & 2.66 \\
\hline 100 & 7.3 & 2.12 & 2.47 & 0.254 & 96.8 & 0.358 & 2.6 \\
\hline
\end{tabular}


Table 3. Performance Metrics of 8-bit MOSFET adder in CPL logic

\begin{tabular}{|c|c|c|c|c|c|c|c|}
\hline Temperature & & All 0's & & All 1's & & & \\
\hline & $\begin{array}{c}\text { Dynamic } \\
\text { Power }\end{array}$ & $\begin{array}{l}\text { Static } \\
\text { Power }\end{array}$ & $\begin{array}{c}\text { Leakage } \\
\text { Power }\end{array}$ & $\begin{array}{l}\text { Static } \\
\text { Power }\end{array}$ & $\begin{array}{c}\text { Leakage } \\
\text { Power }\end{array}$ & Delay & $\begin{array}{c}\text { Power Delay } \\
\text { Product }\end{array}$ \\
\hline (Celsius) & $(\mu \mathrm{W})$ & $(\mathrm{mW})$ & $(\mu \mathrm{W})$ & $(\mu \mathrm{W})$ & $(\mu \mathrm{W})$ & $(\mu s)$ & \\
\hline-25 & 882.6 & 1.68 & 139.9 & 945.76 & 134.7 & 0.46 & 405.99 \\
\hline 0 & 807.3 & 1.59 & 147.88 & 752.3 & 119.8 & 0.457 & 368.99 \\
\hline 25 & 727.56 & 1.25 & 151.98 & 678.65 & 108.6 & 0.45 & 327.41 \\
\hline 50 & 643.28 & 1.1 & 149.7 & 605.1 & 98.03 & 0.449 & 283.04 \\
\hline 75 & 587.71 & 1.05 & 98.88 & 543.8 & 88.15 & 0.448 & 263.2 \\
\hline 100 & 541.14 & 1.03 & 48.54 & 491.4 & 80.43 & 0.443 & 239.7 \\
\hline 125 & 496.56 & 1.006 & 21.76 & 446.2 & 74.78 & 0.44 & 218.05 \\
\hline
\end{tabular}

Table 4. Performance metrics of 8-bit MOSFET adder in TG logic

\begin{tabular}{|c|c|c|c|c|c|c|c|}
\hline Temperature & & All 0's & & All 1's & & & \\
\hline & $\begin{array}{c}\text { Dynamic } \\
\text { Power }\end{array}$ & $\begin{array}{l}\text { Static } \\
\text { Power }\end{array}$ & $\begin{array}{c}\text { Leakage } \\
\text { Power }\end{array}$ & $\begin{array}{l}\text { Static } \\
\text { Power }\end{array}$ & $\begin{array}{c}\text { Leakage } \\
\text { Power }\end{array}$ & Delay & $\begin{array}{c}\text { Power Delay } \\
\text { Product }\end{array}$ \\
\hline (Celsius) & $(\mu \mathrm{W})$ & $(\mu \mathrm{W})$ & $(\mu \mathrm{W})$ & $(\mu \mathrm{W})$ & $(\mu \mathrm{W})$ & $(\mu s)$ & \\
\hline-25 & 11.56 & 0.027 & 9.75 & 3.2 & 3.2 & 0.36 & 4.16 \\
\hline
\end{tabular}


Table 4 Continued

\begin{tabular}{|l|l|l|l|l|l|l|l|}
\hline 0 & 16.29 & 0.34 & 14.34 & 4.87 & 5.01 & 0.366 & 5.96 \\
\hline 25 & 22 & 0.38 & 20.33 & 6.68 & 7.1 & 0.38 & 8.36 \\
\hline 50 & 28.55 & 0.44 & 27.02 & 8.91 & 9.75 & 0.385 \\
\hline 75 & 36.07 & 0.877 & 34.26 & 11.28 & 12.81 & 0.4 & 14.42 \\
\hline 100 & 44.57 & 1.53 & 42.06 & 13.93 & 16.57 & 0.408 & 18.18 \\
\hline
\end{tabular}

Table 5. Performance metrics of 8-bit FinFET adder in CMOS logic

\begin{tabular}{|c|c|c|c|c|c|c|c|}
\hline Temperature & & All 0's & & All 1's & & & \\
\hline & $\begin{array}{c}\text { Dynamic } \\
\text { Power }\end{array}$ & $\begin{array}{l}\text { Static } \\
\text { Power }\end{array}$ & $\begin{array}{c}\text { Leakage } \\
\text { Power }\end{array}$ & $\begin{array}{l}\text { Static } \\
\text { Power }\end{array}$ & $\begin{array}{c}\text { Leakage } \\
\text { Power }\end{array}$ & Delay & $\begin{array}{c}\text { Power Delay } \\
\text { Product }\end{array}$ \\
\hline (Celsius) & $(\mathbf{n W})$ & $(\mathbf{n W})$ & $(\mathbf{n W})$ & $(\mathrm{pW})$ & $(\mathbf{n W})$ & $(\mu s)$ & \\
\hline-25 & 2.98 & 0.226 & 1.121 & 0.725 & 1.69 & 0.28 & 0.83 \\
\hline 0 & 4.08 & 0.278 & 1.889 & 5.79 & 2.55 & 0.26 & 1.06 \\
\hline 25 & 5.41 & 0.527 & 2.708 & 37.67 & 3.56 & 0.25 & 1.35 \\
\hline 50 & 7.01 & 0.762 & 3.78 & 92.13 & 4.72 & 0.22 & 1.54 \\
\hline 75 & 8.81 & 1.01 & 5.116 & 113.17 & 6.054 & 0.19 & 1.67 \\
\hline 100 & 10.87 & 1.338 & 6.65 & 151.67 & 7.63 & 0.18 & 1.95 \\
\hline 125 & 13.27 & 1.671 & 8.581 & 198.7 & 9.536 & 0.15 & 1.99 \\
\hline
\end{tabular}


Table 6. Performance metrics of 8-bit FinFET adder in GDI logic

\begin{tabular}{|c|c|c|c|c|c|c|c|}
\hline Temperature & & All 0's & & All 1's & & & \\
\hline & $\begin{array}{c}\text { Dynamic } \\
\text { Power }\end{array}$ & $\begin{array}{l}\text { Static } \\
\text { Power }\end{array}$ & $\begin{array}{c}\text { Leakage } \\
\text { Power }\end{array}$ & $\begin{array}{l}\text { Static } \\
\text { Power }\end{array}$ & $\begin{array}{c}\text { Leakage } \\
\text { Power }\end{array}$ & Delay & $\begin{array}{c}\text { Power Delay } \\
\text { Product }\end{array}$ \\
\hline (Celsius) & $(\mathbf{n W})$ & $(\mathrm{pW})$ & $(\mathbf{n W})$ & $(\mathrm{pW})$ & $(\mathrm{pW})$ & $(\mu s)$ & \\
\hline-25 & 4.04 & 0.27 & 0.15 & 7.31 & 0.368 & 0.235 & 0.94 \\
\hline 0 & 2.94 & 0.069 & 0.23 & 9.47 & 1.18 & 0.239 & 0.71 \\
\hline 25 & 2.53 & 0.034 & 0.33 & 14.69 & 3.2 & 0.24 & 9.61 \\
\hline 50 & 2.28 & 2.01 & 0.45 & 25.47 & 7.38 & 0.243 & 0.55 \\
\hline 75 & 2.17 & 1.393 & 0.68 & 44.92 & 14.76 & 0.2477 & 0.53 \\
\hline 100 & 2.15 & 0.626 & 0.72 & 75.51 & 28.62 & 0.248 & 0.529 \\
\hline 125 & 2.189 & 7.59 & 0.89 & 133.51 & 46.66 & 0.25 & 0.54 \\
\hline
\end{tabular}

Table 7. Performance metrics of 8-bit FinFET adder in CPL logic

\begin{tabular}{|c|c|c|c|c|c|c|c|}
\hline Temperature & & All 0’s & & All 1's & & & \\
\hline & $\begin{array}{l}\text { Dynamic } \\
\text { Power }\end{array}$ & $\begin{array}{l}\text { Static } \\
\text { Power }\end{array}$ & $\begin{array}{c}\text { Leakage } \\
\text { Power }\end{array}$ & $\begin{array}{l}\text { Static } \\
\text { Power }\end{array}$ & $\begin{array}{c}\text { Leakage } \\
\text { Power }\end{array}$ & Delay & $\begin{array}{c}\text { Power Delay } \\
\text { Product }\end{array}$ \\
\hline (Celsius) & $(\mathrm{nW})$ & $(\mathrm{nW})$ & $(\mathrm{nW})$ & $(\mathrm{nW})$ & $(\mathrm{nW})$ & $(\mu s)$ & \\
\hline-25 & 156.2 & 305.3 & 25.77 & 170.4 & 24.77 & 0.19 & 29.67 \\
\hline
\end{tabular}


Table 3 Continued

\begin{tabular}{|c|c|c|c|c|c|c|c|}
\hline 0 & 142 & 291.1 & 27.19 & 134.9 & 22.08 & 0.196 & 27.83 \\
\hline 25 & 127.8 & 227.2 & 27.69 & 120.7 & 19.8 & 0.2 & 25.56 \\
\hline 50 & 113.6 & 198.8 & 27.33 & 106.5 & 17.89 & 0.21 & 23.86 \\
\hline 75 & 106.5 & 191.7 & 18.17 & 99.4 & 16.25 & 0.22 & 23.43 \\
\hline 100 & 99.4 & 184.6 & 8.73 & 85.2 & 14.83 & 0.225 & 22.36 \\
\hline 125 & 85.2 & 177.5 & 3.9 & 78.1 & 13.63 & 0.23 & 19.59 \\
\hline
\end{tabular}

Table 8. Performance metrics of 8-bit FinFET adder in TG logic

\begin{tabular}{|c|c|c|c|c|c|c|c|}
\hline Temperature & & All 0's & & All 1's & & & \\
\hline & $\begin{array}{c}\text { Dynamic } \\
\text { Power }\end{array}$ & $\begin{array}{l}\text { Static } \\
\text { Power }\end{array}$ & $\begin{array}{c}\text { Leakage } \\
\text { Power }\end{array}$ & $\begin{array}{l}\text { Static } \\
\text { Power }\end{array}$ & $\begin{array}{c}\text { Leakage } \\
\text { Power }\end{array}$ & Delay & $\begin{array}{c}\text { Power Delay } \\
\text { Product }\end{array}$ \\
\hline (Celsius) & $(\mathrm{nW})$ & $(\mathrm{nW})$ & $(\mathrm{nW})$ & $(\mathrm{nW})$ & $(\mathrm{nW})$ & $(\mu s)$ & \\
\hline-25 & 52.1 & 0.125 & 44 & 14.65 & 14.87 & 0.222 & 11.4 \\
\hline 0 & 73.45 & 1.622 & 64.67 & 21.95 & 22.57 & 0.225 & 16.52 \\
\hline 25 & 99.1 & 2.03 & 92.02 & 30.57 & 32.25 & 0.23 & 22.79 \\
\hline 50 & 128.95 & 2.037 & 121.7 & 40.5 & 43.95 & 0.24 & 30.94 \\
\hline 75 & 162.82 & 3.97 & 154.42 & 51.25 & 58 & 0.243 & 39.56 \\
\hline 100 & 200.82 & 7.225 & 189.77 & 62.92 & 74.8 & 0.248 & 49.8 \\
\hline 125 & 244.5 & 13.05 & 227.6 & 76.22 & 95.73 & 0.25 & 61.12 \\
\hline
\end{tabular}




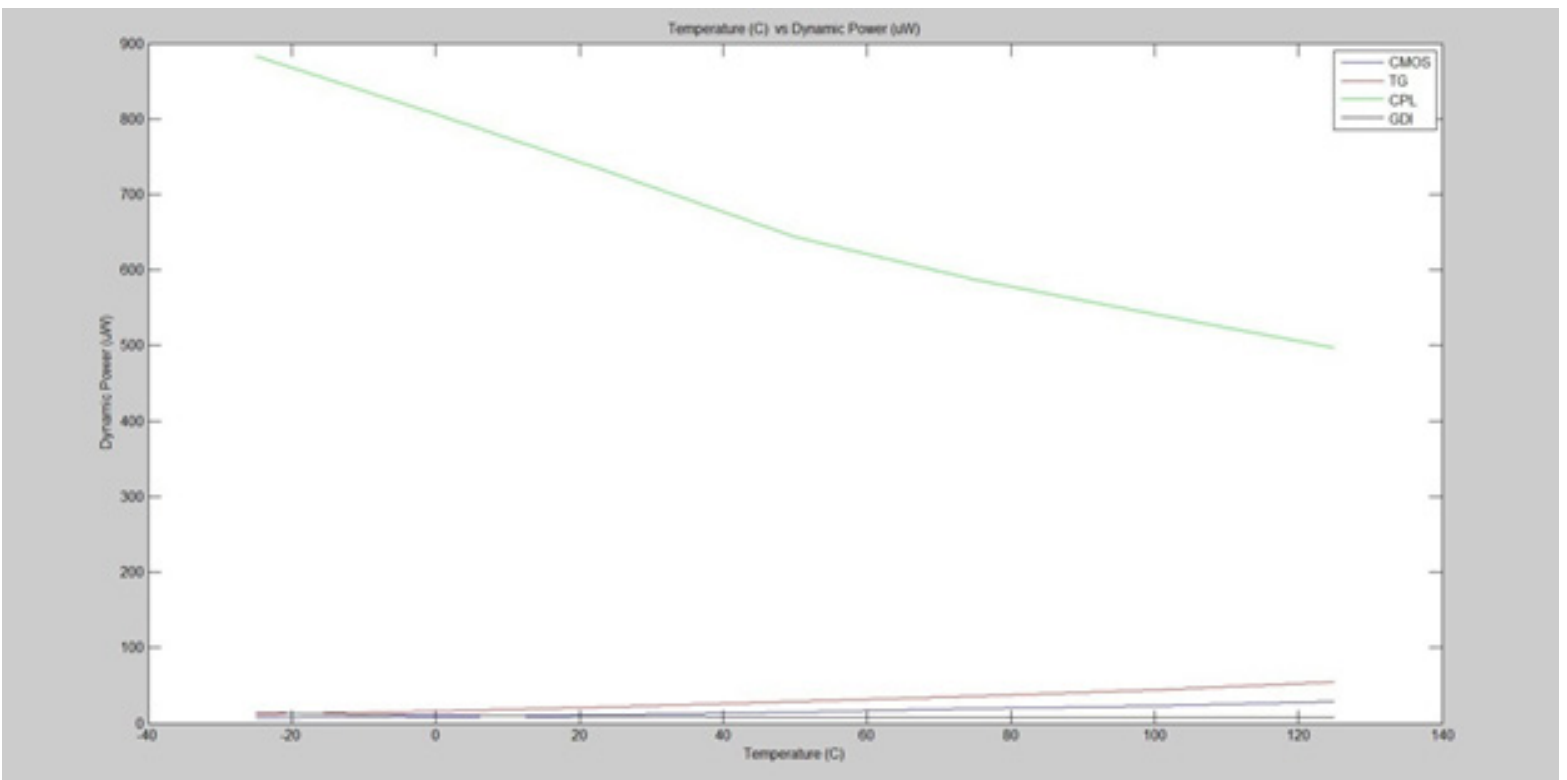

Figure 17. Dynamic power of 8-bit MOSFET adder.
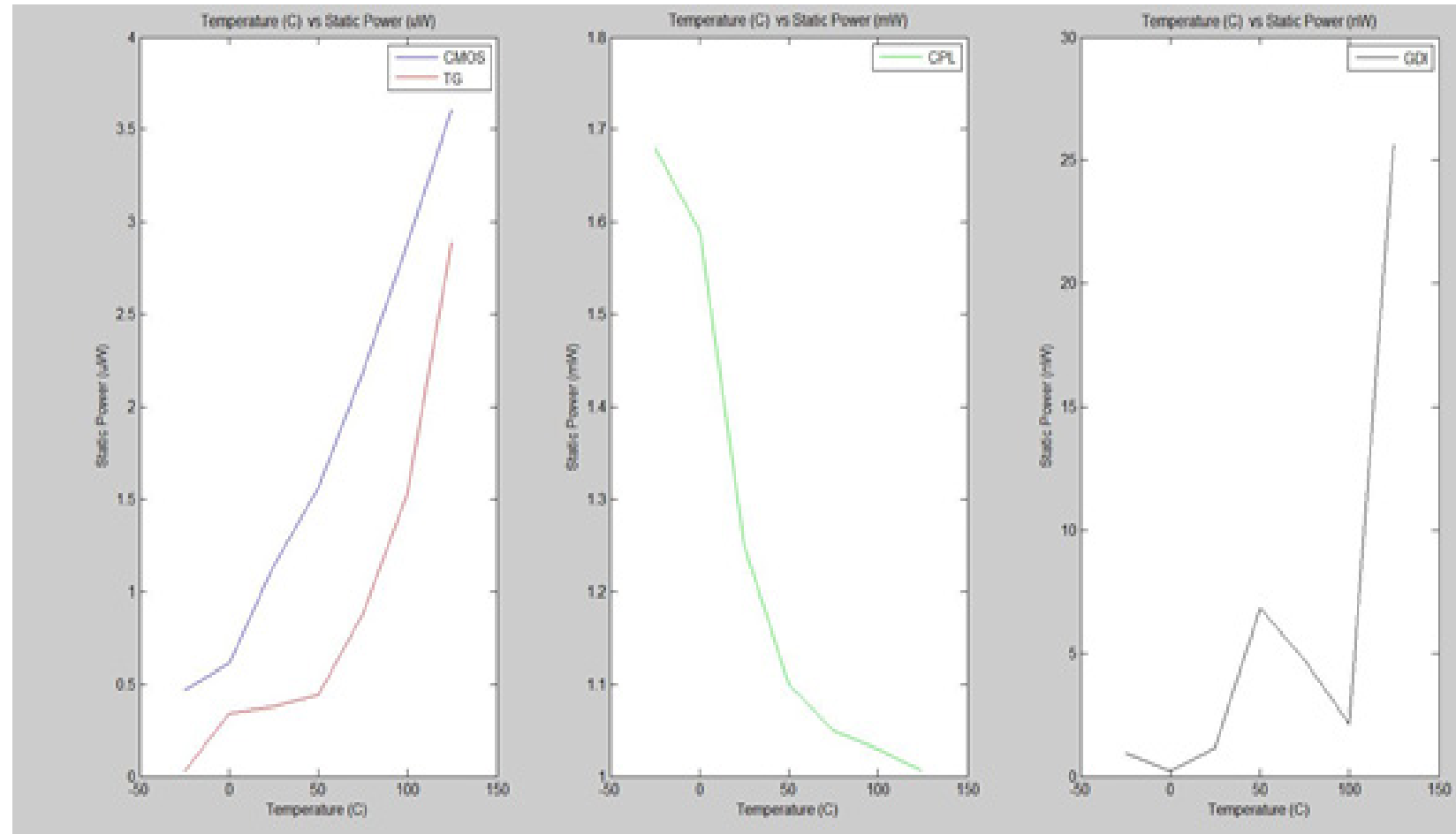

Figure 18. Static power (inputs at low logic level) of 8-bit MOSFET adder.

8-bit Fin FET adder implemented in CPL logic. The Figure 14 represents the Input / Output (I/O) waveform of this adder. Table 7 contains the information about variation of the performance metrics of this adder in accordance to the 


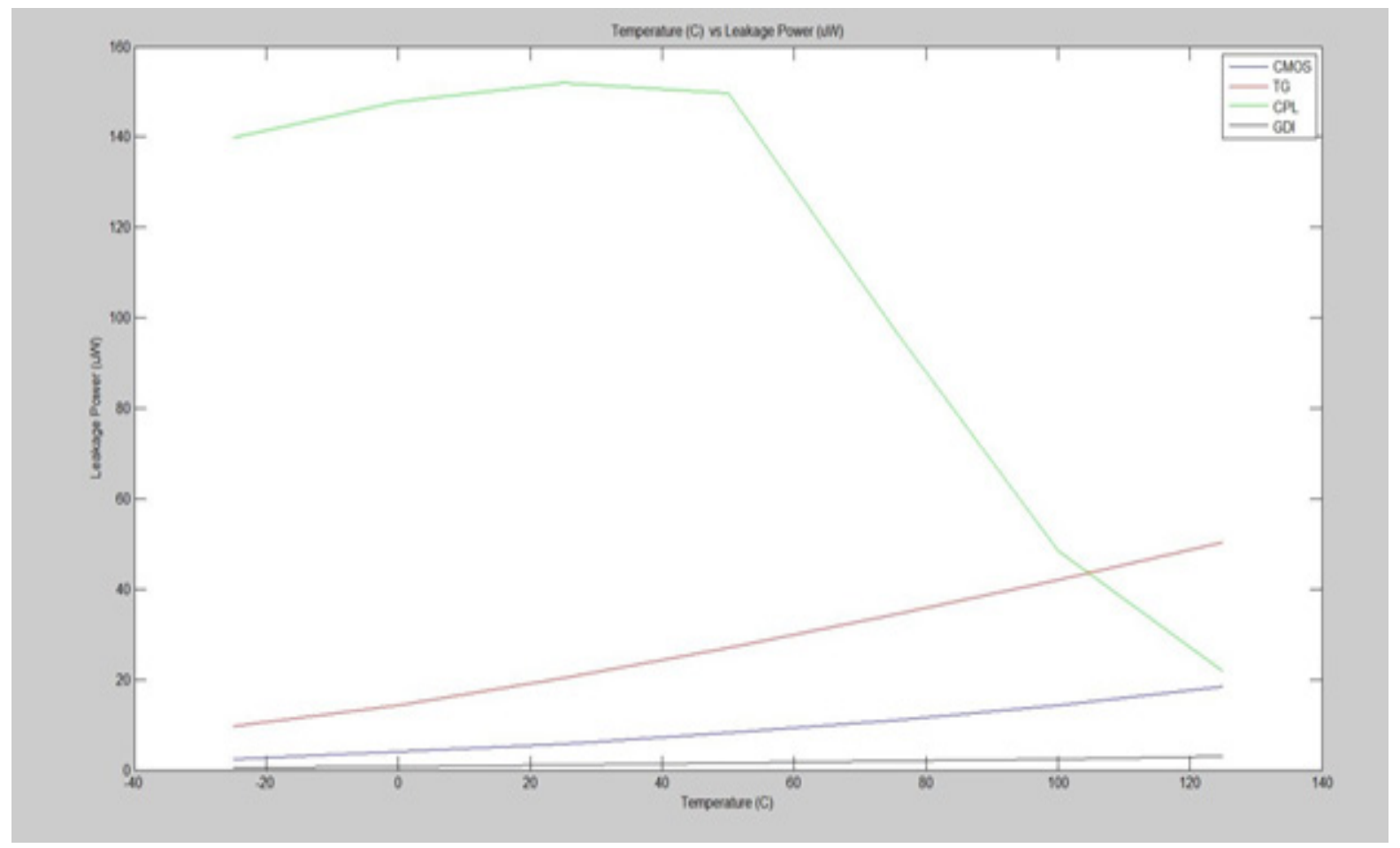

Figure 19. Leakage power (inputs at low logic level) of 8-bit MOSFET adder.

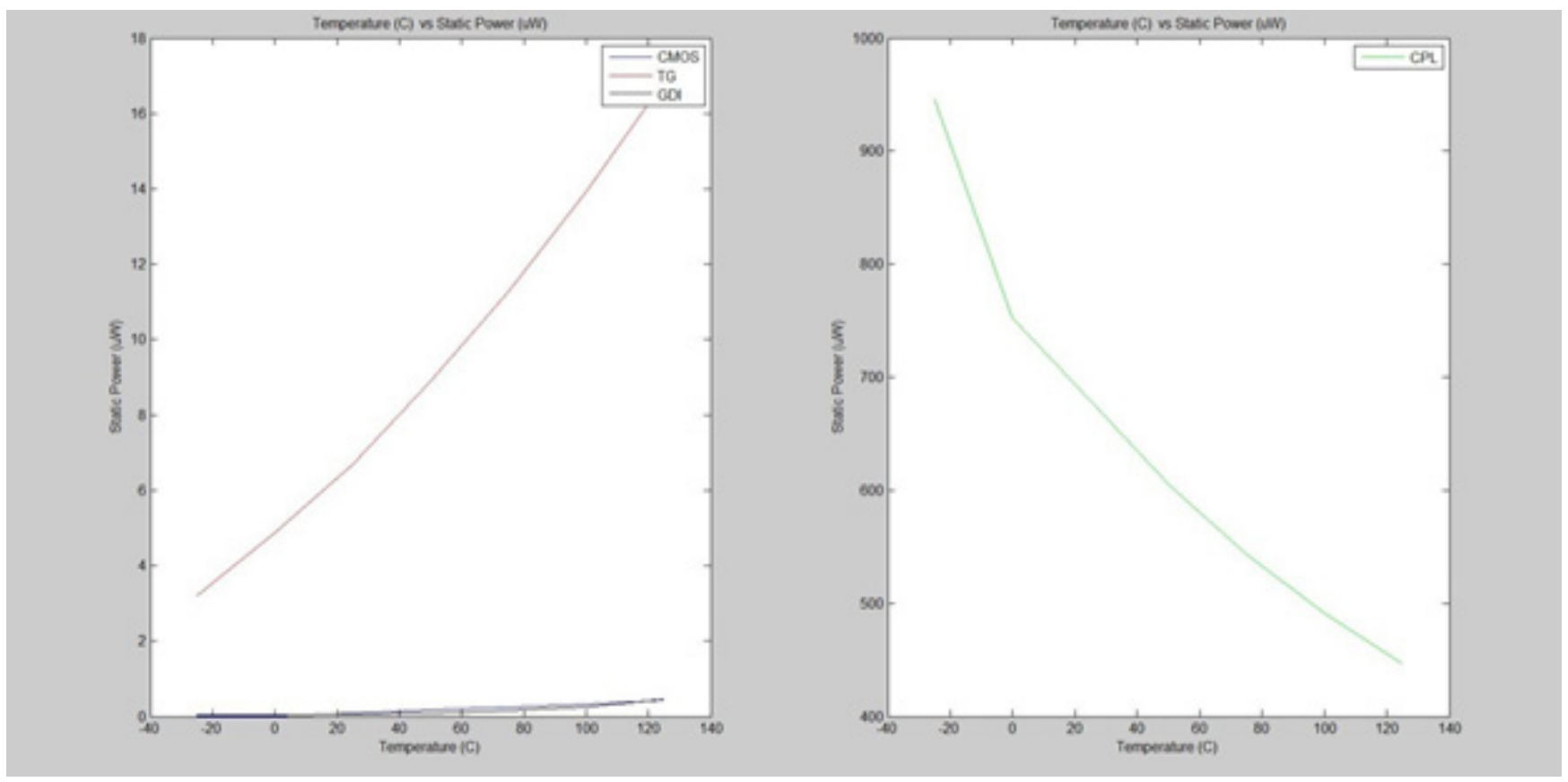

Figure 20. Static power (inputs at high logic level) of 8-bit MOSFET adder.

range of temperatures. Figure 15 depicts the schematic of 8 -bit Fin FET adder implemented in TG logic. The Figure
16 represents the Input / Output $(\mathrm{I} / \mathrm{O})$ waveform of this adder. Table 8 contains the information about variation 


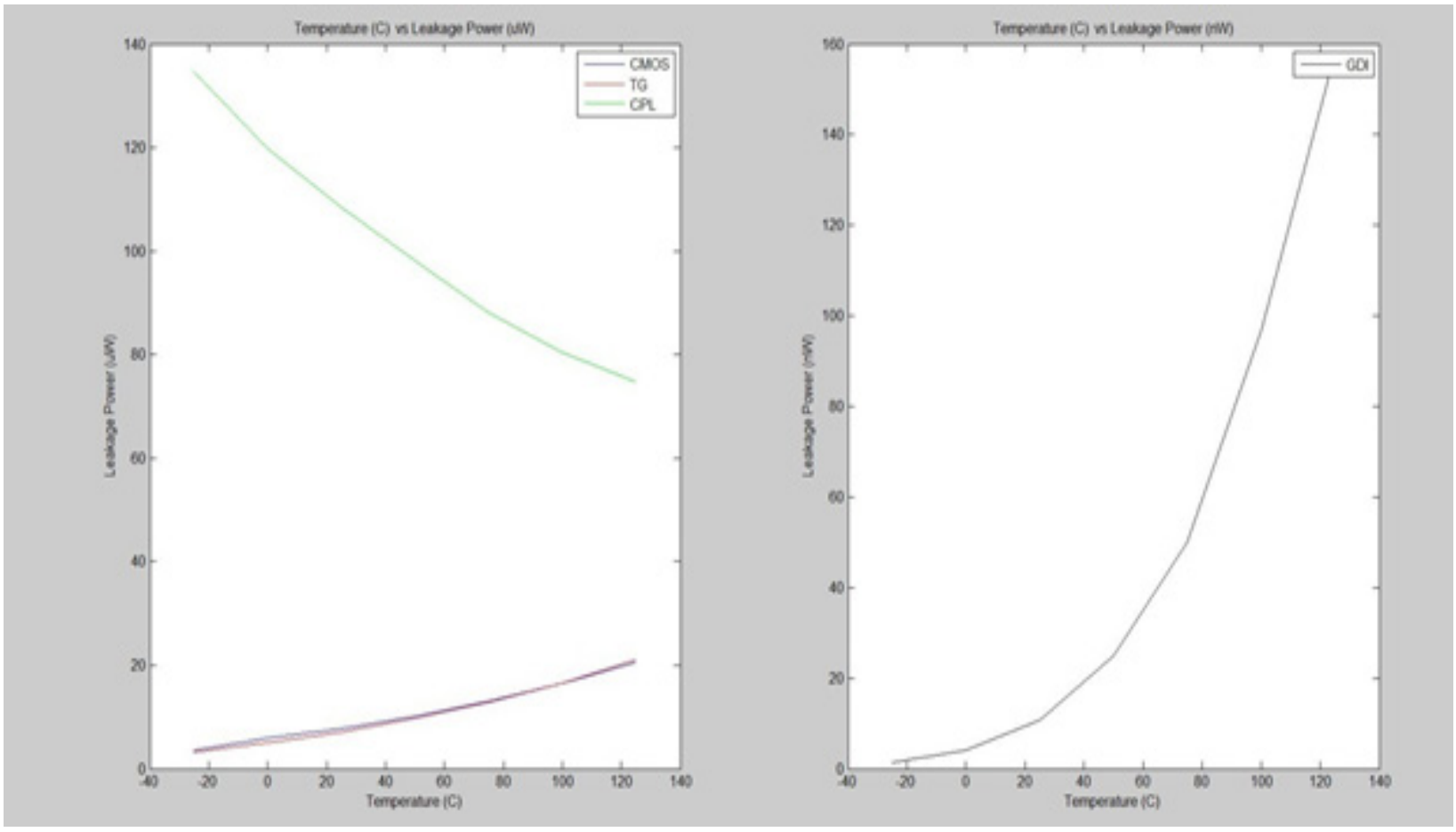

Figure 21. Leakage power (inputs at high logic level) of 8-bit MOSFET adder.

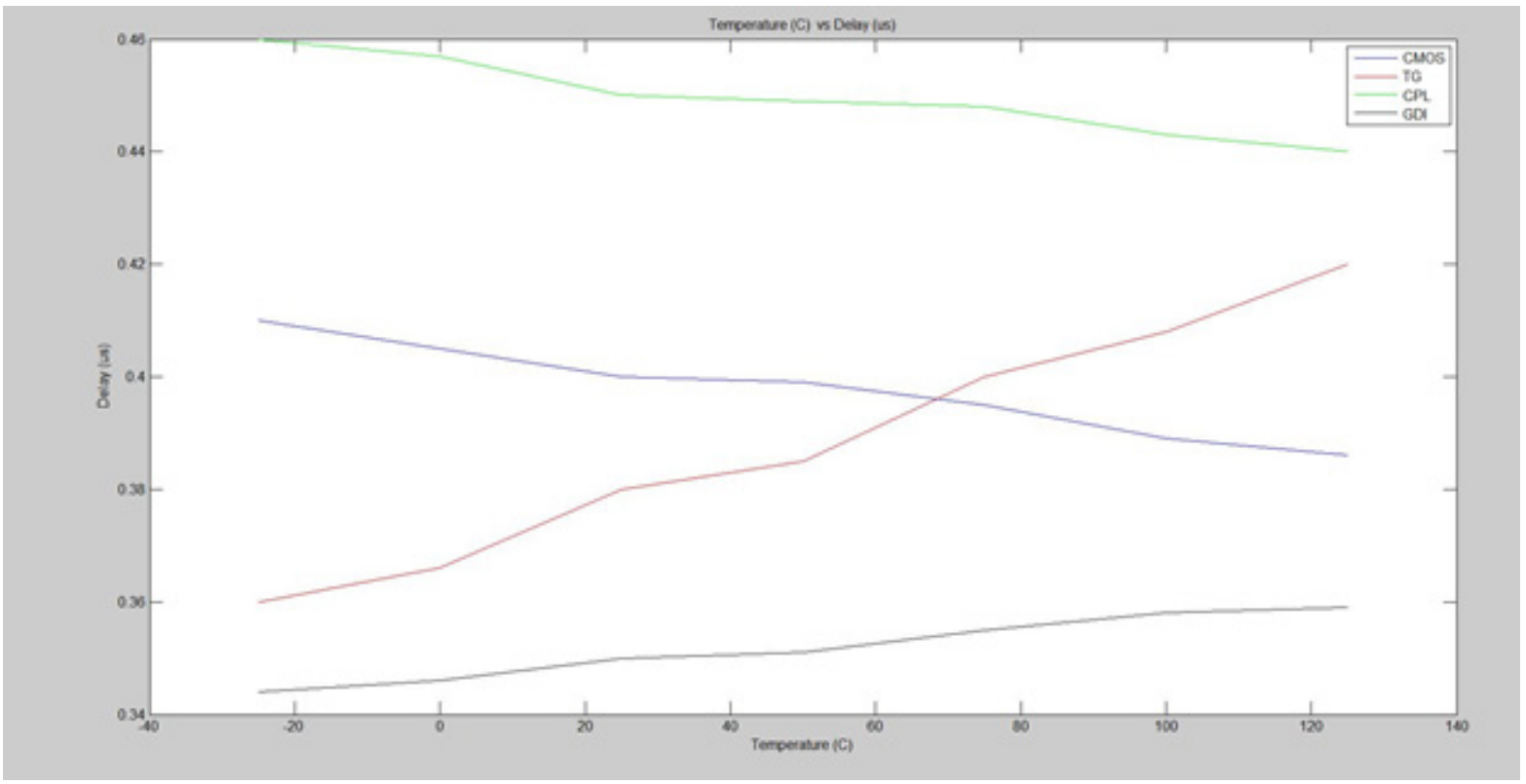

Figure 22. Propagation delay of 8-bit MOSFET adder.

of the performance metrics of this adder in accordance to the range of temperatures.
The dynamic power of 8-bit MOSFET adder (for all logic styles) is correlated with range of temperature val- 


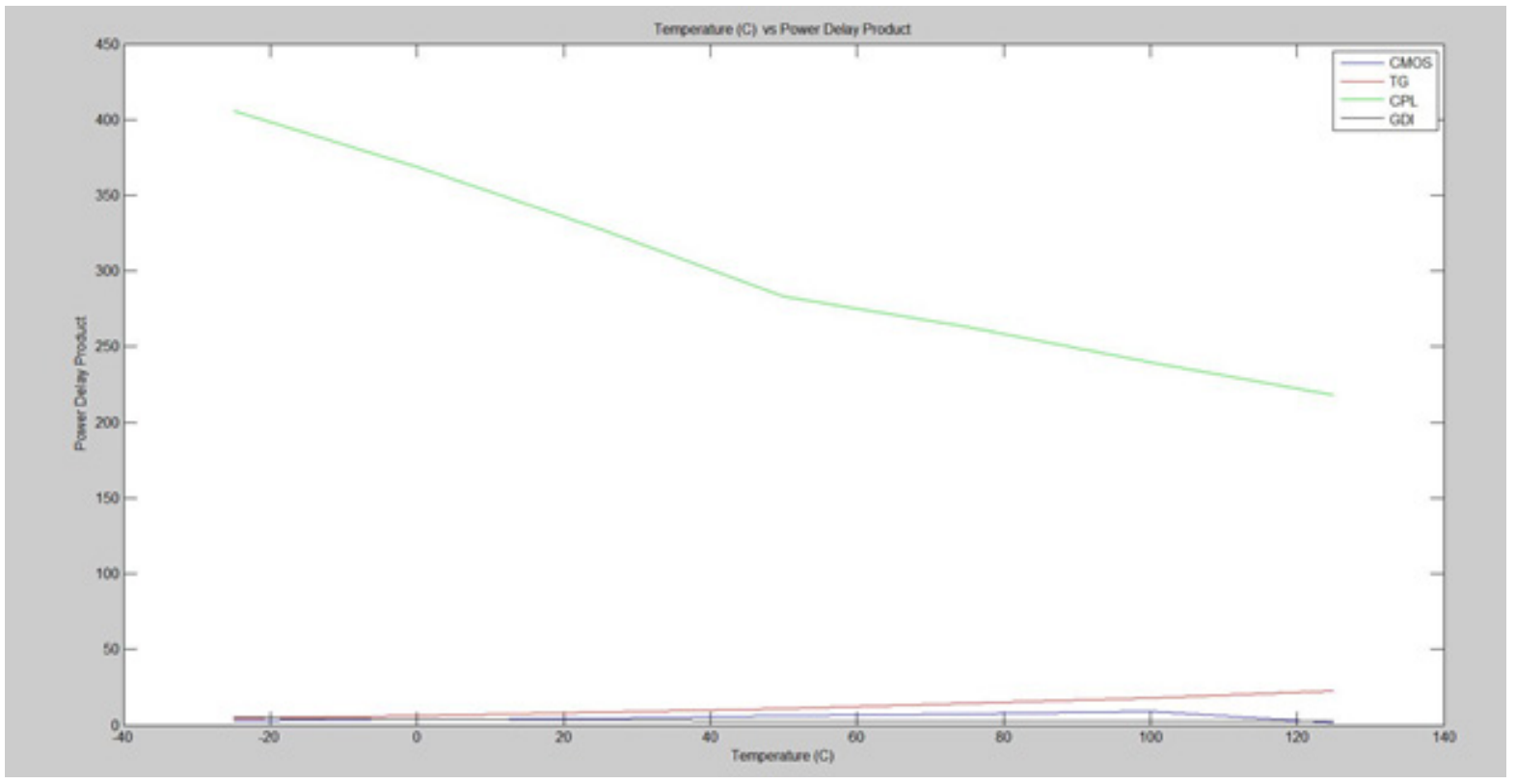

Figure 23. Power delay Product of 8-bit MOSFET adder.

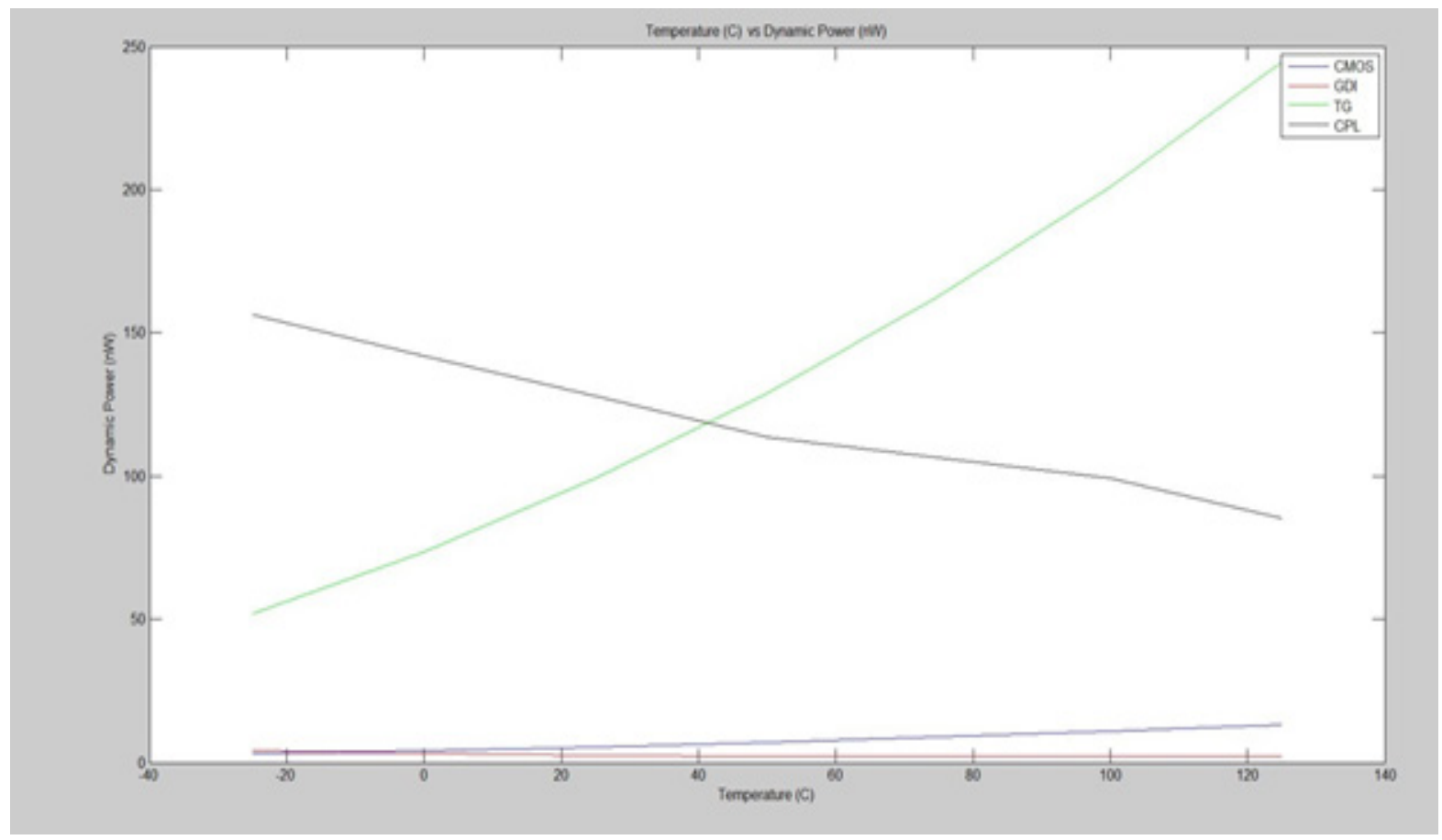

Figure 24. Dynamic power of 8-bit FinFET adder.

ues, is graphically represented in Figure 17. The static power (inputs at low logic level) of 8-bit MOSFET adder (for all logic styles) is correlated with range of temperature values, is graphically represented in Figure 18. The 


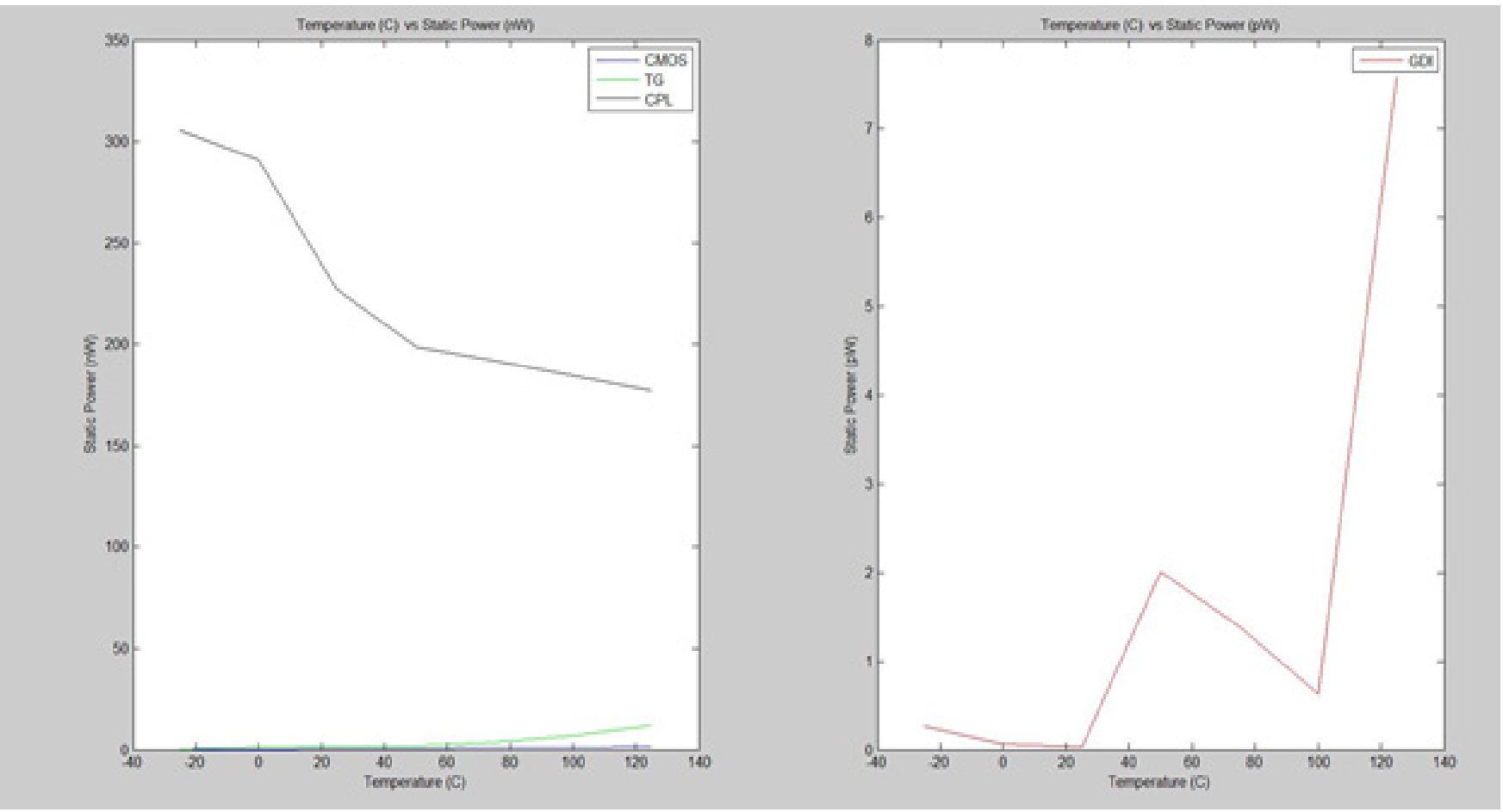

Figure 25. Static power (inputs at low logic level) of 8-bit FinFET adder.

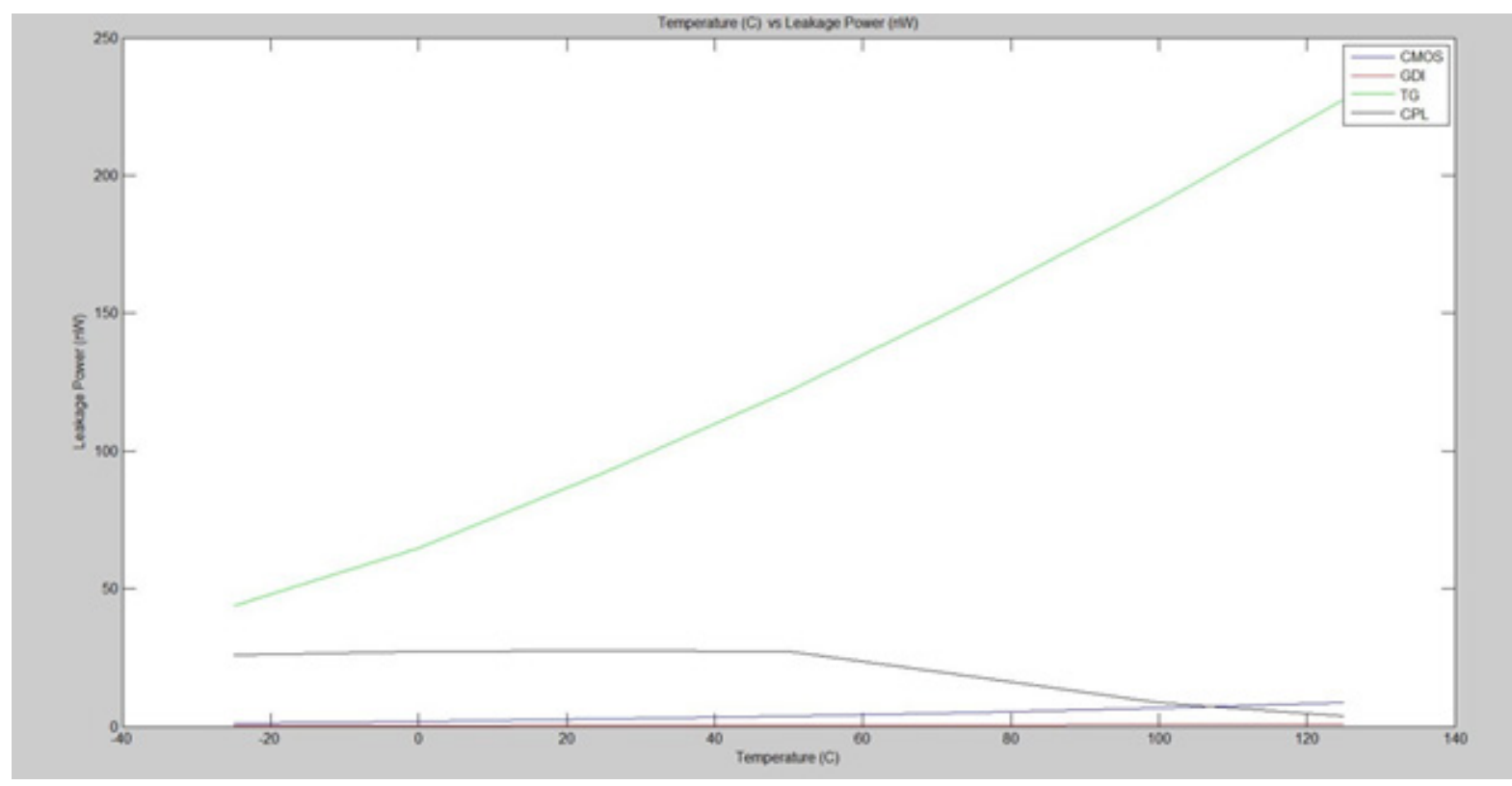

Figure 26. Leakage power (inputs at low logic level) of 8-bit FinFET adder.

leakage power (inputs at low logic level) of 8-bit MOSFET adder (for all logic styles) is correlated with range of temperature values, is graphically represented in Figure 19. The static power (inputs at high logic level) of 8-bit 

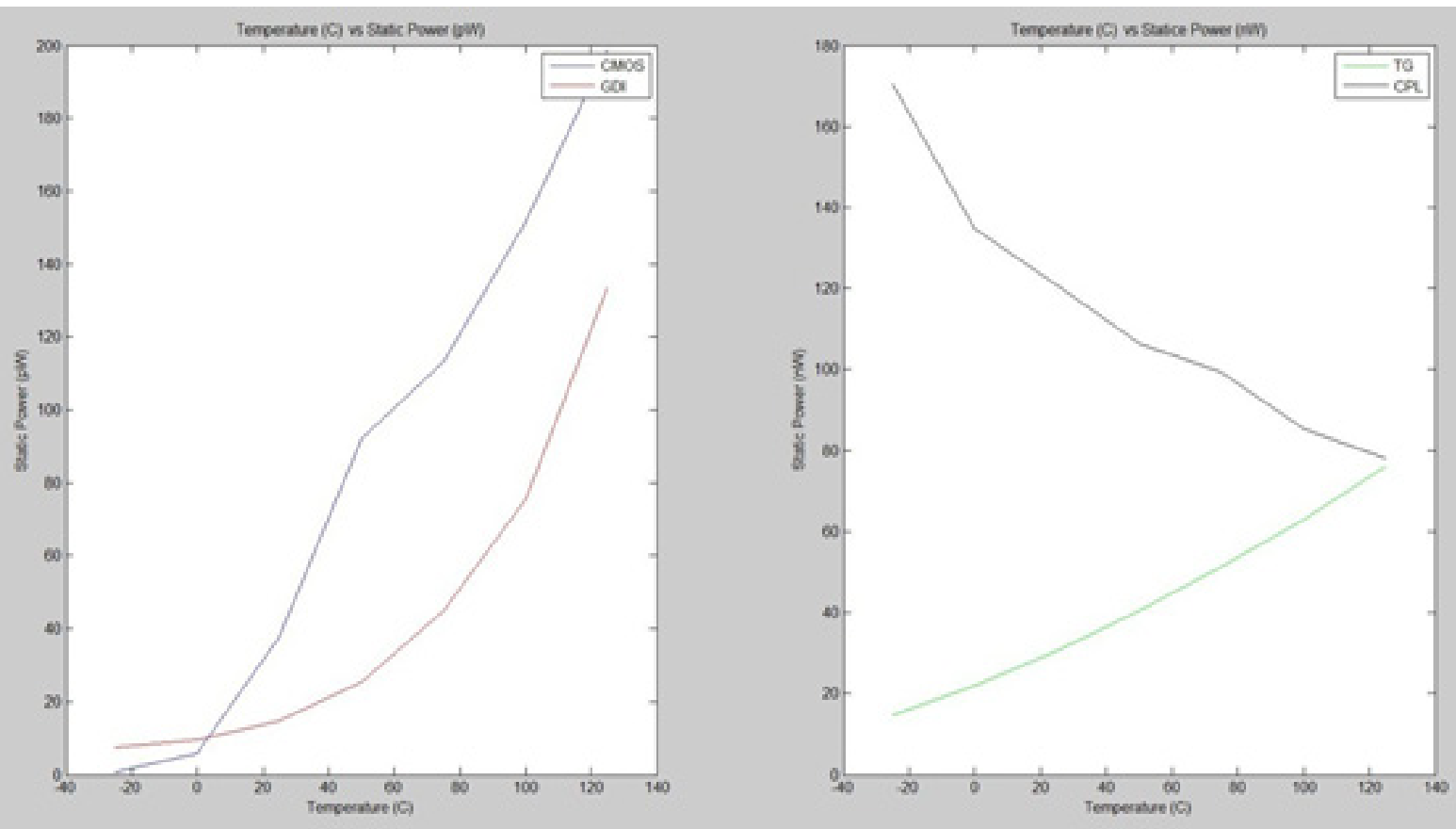

Figure 27. Static power (inputs at high logic level) of 8-bit FinFET adder.

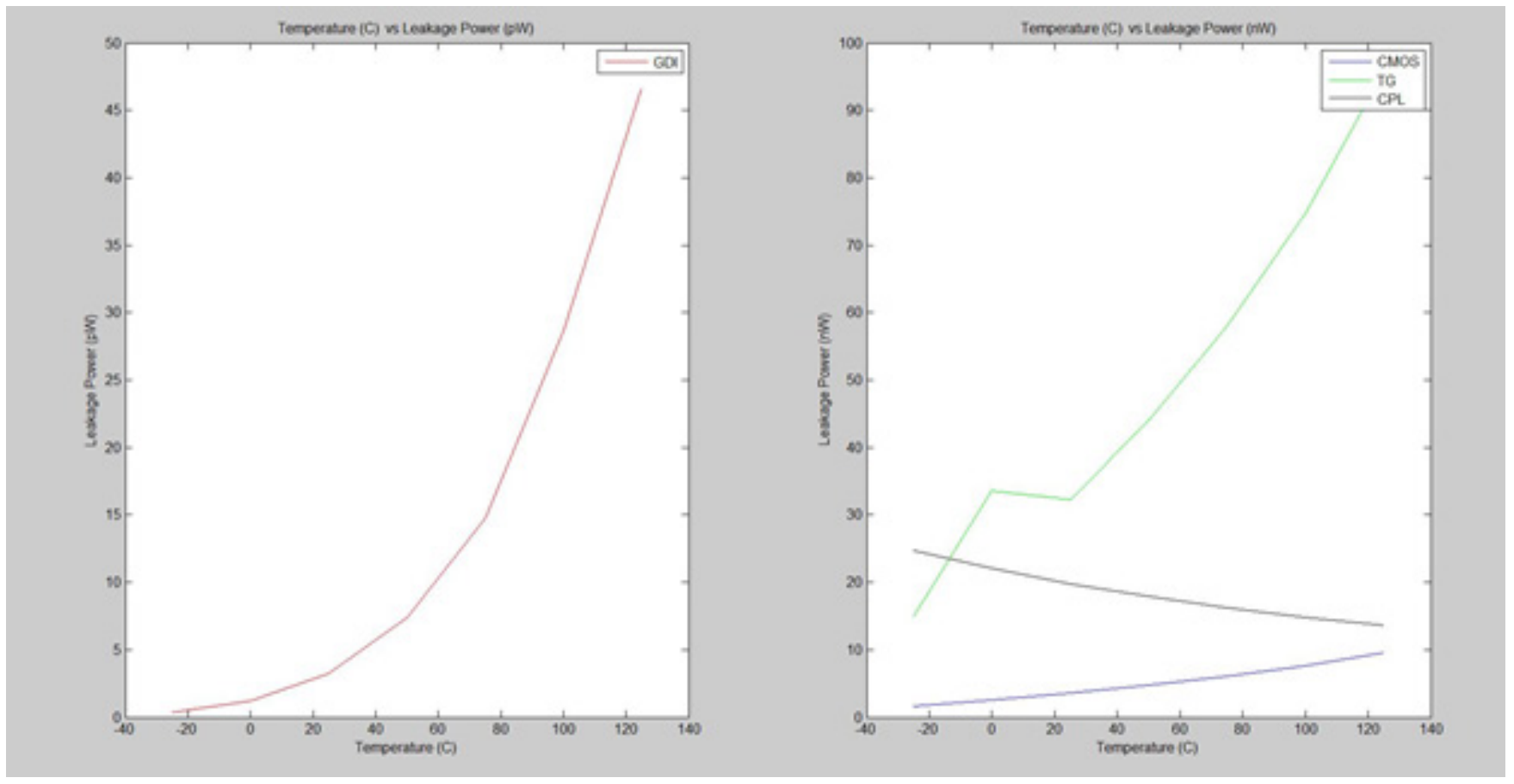

Figure 28. Leakage power (inputs at high logic level) of 8-bit FinFET adder.

MOSFET adder (for all logic styles) is correlated with range of temperature values, is graphically represented in
Figure 20. The leakage power (inputs at high logic level) of 8-bit MOSFET adder (for all logic styles) is correlated 


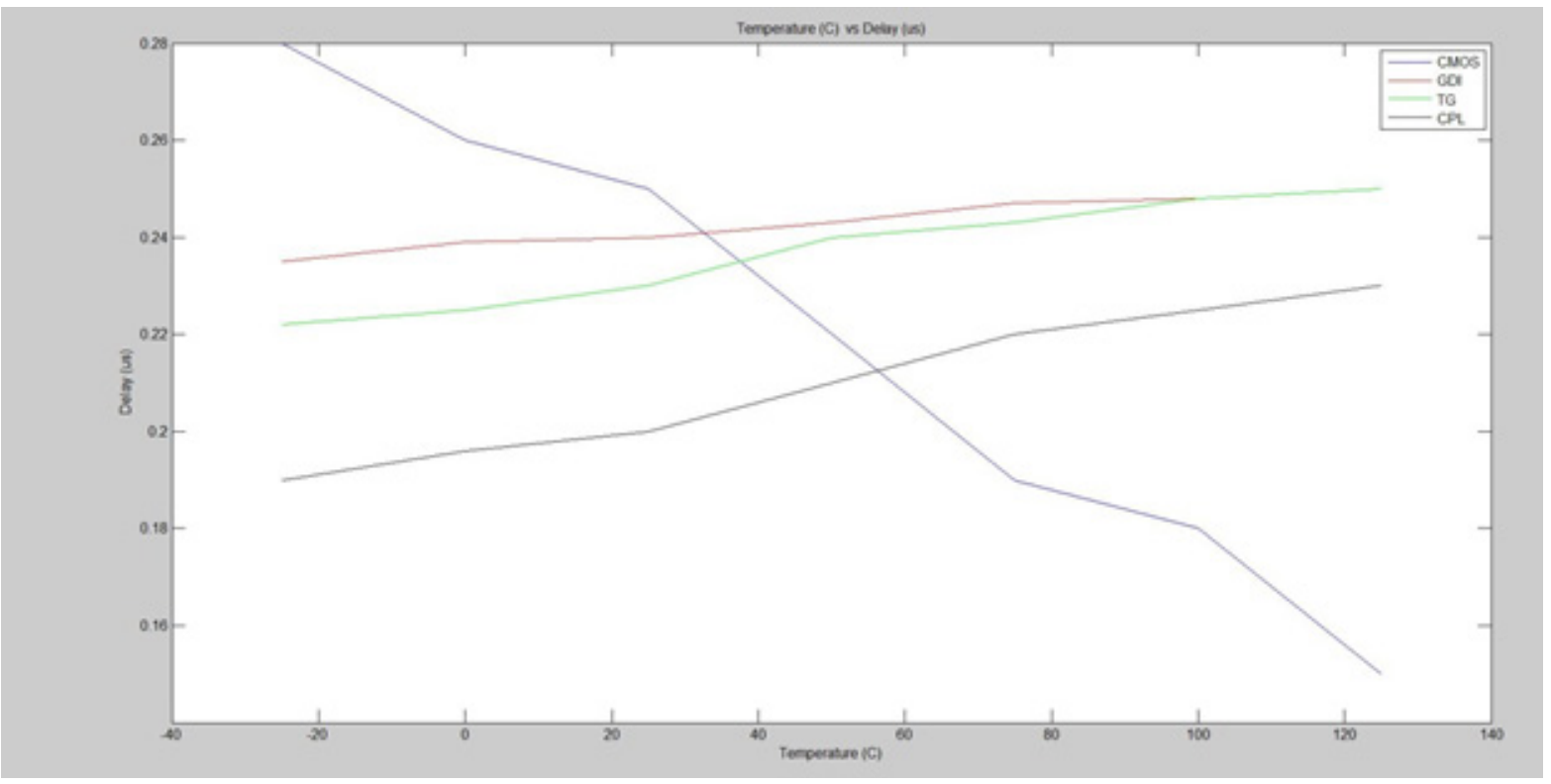

Figure 29. Delay of 8-bit FinFET adder.

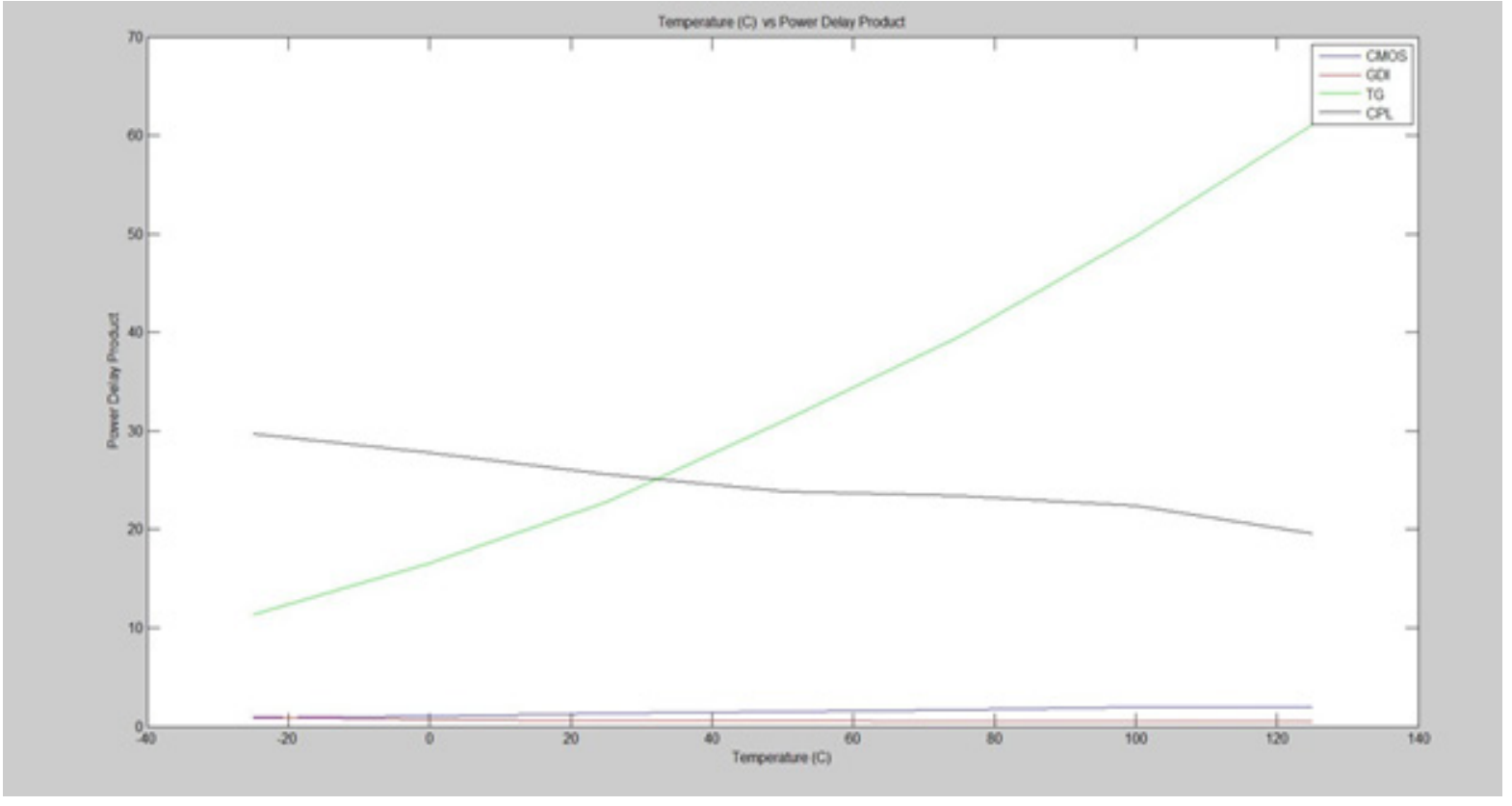

Figure 30. Power delay Product of 8-bit FinFET adder.

with range of temperature values, is graphically represented in Figure 21. The delay of 8-bit MOSFET adder (for all logic styles) is correlated with range of tempera- ture values, is graphically represented in Figure 22. The power delay product of 8-bit MOSFET adder (for all logic 
styles) is correlated with range of temperature values, is graphically represented in Figure 23.

The dynamic power of 8-bit Fin FET adder (for all logic styles) is correlated with range of temperature values, is graphically represented in Figure 24. The static power (inputs at low logic level) of 8-bit Fin FET adder (for all logic styles) is correlated with range of temperature values, is graphically represented in Figure 25. The leakage power (inputs at low logic level) of 8-bit Fin FET adder (for all logic styles) is correlated with range of temperature values, is graphically represented in Figure 26. The static power (inputs at high logic level) of 8-bit Fin FET adder (for all logic styles) is correlated with range of temperature values, is graphically represented in Figure 27. The leakage power (inputs at high logic level) of 8-bit Fin FET adder (for all logic styles) is correlated with range of temperature values, is graphically represented in Figure 28. The delay of 8-bit Fin FET adder (for all logic styles) is correlated with range of temperature values, is graphically represented in Figure 29. The power delay product of 8-bit Fin FET adder (for all logic styles) is correlated with range of temperature values, is graphically represented in Figure 30.

Figure 17 and 24 we can infer that the maximum dynamic power dissipated by an 8-bit MOSFET adder is $0.882 \mathrm{~mW}$ and that of an 8 -bit Fin FET adder is $0.156 \mu \mathrm{W}$. From Figure 18 and 25 we can infer that the maximum static power (inputs at low logic level) dissipated by an 8 -bit MOSFET adder is $1.68 \mathrm{~mW}$ and that of an 8 -bit Fin FET adder is $0.305 \mu W$. From Figure 19 and 26 we can infer that the maximum leakage power (inputs at low logic level) dissipated by an 8 -bit MOSFET adder is $0.139 \mathrm{~mW}$ and that of an 8-bit Fin FET adder is $0.227 \mu \mathrm{W}$. From Figure 20 and 27 we can infer that the maximum static power (inputs at high logic level) dissipated by an 8-bit MOSFET adder is $0.945 \mathrm{~mW}$ and that of an 8-bit Fin FET adder is $0.17 \mu \mathrm{W}$. From Figure 21 and 28 we can infer that the maximum leakage power (inputs at high logic level) dissipated by an 8 -bit MOSFET adder is $0.134 \mathrm{~mW}$ and that of an 8-bit Fin FET adder is $0.024 \mu \mathrm{W}$. From Figure 22 and 29 we can infer that the maximum propagation delay value of an 8-bit MOSFET adder is $0.46 \mu$ s and that of an 8 -bit Fin FET adder is $0.28 \mu$ s. From Figure 23 and 30 we can infer that the maximum power delay product of an 8 -bit MOSFET adder is $0.405 \mathrm{pJ}$ and that of an 8 -bit Fin FET adder is $0.029 \mathrm{pJ}$.

\section{Conclusion}

FINFET not only has exceptional performance over MOSFET but is prepped to take over MOSFET as the superior technology below $90 \mathrm{~nm}$. The modeling and optimization of the adder, was done at $32 \mathrm{~nm}$ technology. The FINFET structure has been investigated with the aim of reducing the leakage power and propagation delay. The FINFET adder gave rise to a reduction in the leakage power dissipation by $99.9 \%$ in addition to a reduced propagation delay by $39 \%$ and power delay product by $29 \%$. It is also evident that the adder implemented in GDI logic style consumes less power than other adder structures.

\section{References}

1. Sharma S, Soni G. Comparison analysis of Fin FET based 1-bit full adder cell implemented using different logic styles at 10, 22 and $32 \mathrm{~nm}$. International Conference on Energy Efficient Technologies for Sustainability (ICEETS); 2016. p. 660-7. Crossref. PMid:27704370 PMCid: PMC5306072

2. Saraswat R, Akashe S, Babu S. Designing and simulation of full adder cell using FINFET technique. 7th International Conference on Intelligent Systems and Control (ISCO); 2013. p. 261-4. Crossref.

3. Jafari M, Imani M, Ansari M, Fathipour M, Sehatbakhsh N. Design of an ultra-Low Power 32-bit Adder Operating at Sub threshold Voltages in 45-nm Fin FET. 8th International Conference on Design and Technology of Integrated Systems in Nanoscale Era (DTIS); 2013. p. 167-8.

4. Tahrim AA, Tan MLP. Design and Implementation of a 1-bit Fin FET Full Adder Cell for ALU in Sub threshold Region. 2014 IEEE International Conference on Semiconductor Electronics (ICSE 2014); 2014. p. 44-7. 
5. Rapolu S, Nikoubin T. Fast and energy efficient Fin FET full adders with cell design methodology (CDM). 6th International Conference on Computing, Communication and Networking Technologies (ICCCNT); 2015. p.1-5.

6. Abbasalizadeh S, Forouzandeh B. Full adder design with GDI cell and independent double gate transistor. 20th Iranian
Conference on Electrical Engineering (ICEE 2012); 2012. p. 130-4. Crossref.

7. Moshgelani F, Al-Khalili D, Rozon C. Adder circuits using symmetric and asymmetric FinFETs. 2nd International Symposium on Instrumentation and Measurement, Sensor Network and Automation (IMSNA); 2013. p. 23-6. Crossref. 